\title{
Multi Sector Planning Tools for Trajectory-Based Operations
}

\author{
Thomas Prevot ${ }^{1}$, Matthew Mainini ${ }^{2}$, and Connie Brasil ${ }^{3}$ \\ San Jose State University/NASA Ames Research Center, Moffett Field, CA, 94035
}

\begin{abstract}
This paper discusses a suite of multi sector planning tools for trajectory-based operations that were developed and evaluated in the Airspace Operations Laboratory (AOL) at the NASA Ames Research Center. The toolset included tools for traffic load and complexity assessment as well as trajectory planning and coordination. The situation assessment tools included an integrated suite of interactive traffic displays, load tables, load graphs, and dynamic aircraft filters. The planning toolset allowed for single and multi aircraft trajectory planning and data communication-based coordination of trajectories between operators. Also newly introduced was a real-time computation of sector complexity into the toolset that operators could use in lieu of aircraft count to better estimate and manage sector workload, especially in situations with convective weather. The tools were used during a joint NASA/FAA multi sector planner simulation in the AOL in 2009 that had multiple objectives with the assessment of the effectiveness of the tools being one of them. Current air traffic control operators who were experienced as area supervisors and traffic management coordinators used the tools throughout the simulation and provided their usefulness and usability ratings in post simulation questionnaires. This paper presents these subjective assessments as well as the actual usage data that was collected during the simulation. The toolset was rated very useful and usable overall. Many elements received high scores by the operators and were used frequently and successfully. Other functions were not used at all, but various requests for new functions and capabilities were received that could be added to the toolset.
\end{abstract}

\section{Nomenclature}

$\begin{array}{ll}\text { AOL } & =\text { Airspace Operations Laboratory at NASA Ames } \\ \text { ANSP } & =\text { Air Navigation Service Provider } \\ D S R & =\text { Display System Replacement } \\ F A A & =\text { Federal Aviation Administration } \\ \text { FBTM } & =\text { Flow-Based Trajectory Management } \\ \text { MACS } & =\text { Multi Aircraft Control System } \\ \text { MSP } & =\text { Multi Sector Planning } \\ \text { NASA } & =\text { National Aeronautics and Space Administration } \\ \text { NextGen } & =\text { Next Generation Air Transportation System } \\ \text { Sup } & =\text { Area Supervisor } \\ T M C & =\text { Traffic Management Coordinator } \\ T M U & =\text { Traffic Management Unit } \\ M A P & =\text { Monitor Alert Parameter }\end{array}$

\footnotetext{
${ }^{1}$ Senior Research Engineer, Human Factors Research and Technology, NASA ARC, MS 262-4, AIAA member.

${ }^{2}$ Research Engineer, Human Factors Research and Technology, NASA ARC, MS 262-4.

${ }^{3}$ Research Engineer, Human Factors Research and Technology, NASA ARC, MS 262-4. 


\section{Introduction}

$\mathrm{T}$

HE Federal Aviation Administration (FAA) and the National Aeronautics and Space Administration (NASA) are jointly investigating new air traffic service provider functions for Multi Sector Planning (MSP). The MSP concept has been proposed to better manage traffic demand and it has been examined both in the U.S. and Europe $1,2,3,4$. The MSP concept introduces an Air Navigation Service Provider (ANSP) position/function that modifies inflight trajectories for aircraft within specific flows, and reduces traffic or airspace complexity to manageable levels across multiple sectors. The potential benefit for such a position or function could be a more responsive and dynamic management of traffic with greater efficiencies relative to current management methods, thereby providing a better distribution of workload and resources at the sector level and reducing impact to system users. The concept was originally developed as a set of functions that would be performed by a new facility position called the MSP. The multi sector planning process includes problem identification, situation assessment, solution development, and plan coordination. Initial identification of the local area problem may occur in the traffic management unit (TMU) or control floor, while situation assessment and plan development may involve traffic management, one or more MSPs, and front line managers depending on the scope and complexity of the problem and its proposed solution. The person(s) who has developed the solution identifies the person(s) impacted by the plan and coordinates with them accordingly. The solution is then sent to the radar sector as a clearance request which the controller reviews and issues to the aircraft if it is acceptable.

Funded by the FAA's Air Traffic Organization for Planning, Research, and Technology, the Airspace Operations Laboratory (AOL) ${ }^{5,6}$ investigated the effectiveness of MSP functions and positions in a series of cognitive walkthroughs and human-in-the-loop studies. In 2009, a joint NASA/FAA simulation had 18 different air traffic control and management positions in Kansas City (ZKC) and Memphis (ZME) ARTCCs (Figure 1) interact with each other and simulation pilots to investigate the MSP concept and the effectiveness of innovative trajectory management tools and functions. In this simulation over 1200 aircraft and complex convective weather was simulated to create challenging problems to the traffic management and air traffic control positions.

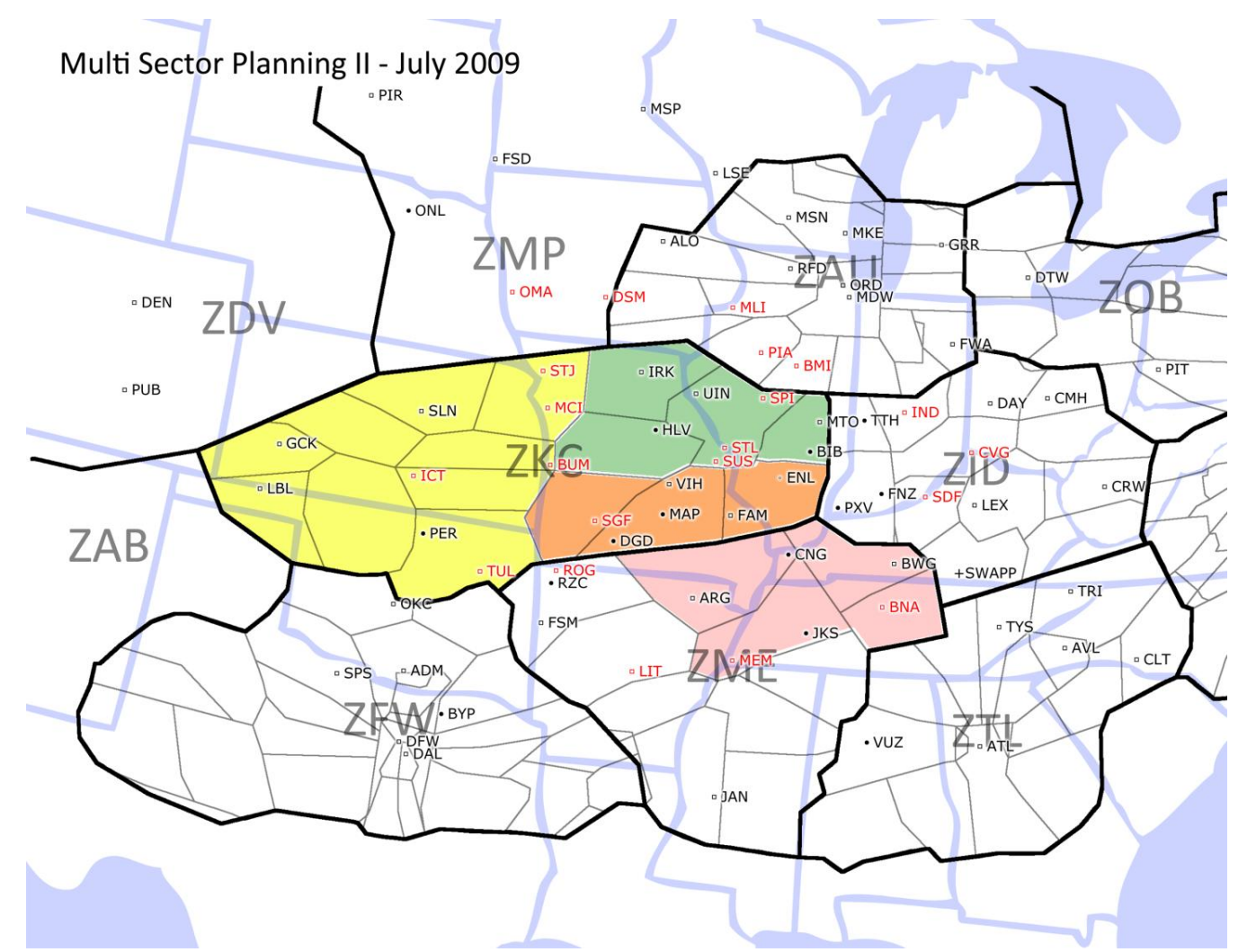

Figure 1. Airspace map for Multi Sector Planner simulation (2009). 
The first goal of the study was to evaluate the effectiveness of a distinct MSP position as compared to distributing MSP functions among existing ANSP positions. The associated findings are presented in a separate publication. ${ }^{2} \mathrm{~A}$ second goal of the study was to evaluate the air traffic management and air traffic control tools used during this simulation. This paper presents the results of this evaluation. The paper is organized as follows: Section II provides an overview of the MSP tools. Section III briefly reviews the experiment design that provided the data for this paper. Section IV shows summary results. Section V presents results on individual tool elements with regard to subjective usability and usefulness data and objective usage data gathered during and after the experiment. Section VI describes requested future features and Section VII presents conclusions.

\section{Multi Sector Planning (MSP) Tools Overview}

The MSP tools were implemented into the AOLs research software, the Multi Aircraft Control System (MACS) ${ }^{7}$ and were designed for trajectory-based operations within the envisioned FAA's mid-term high altitude environment ${ }^{8}$. The tools were built upon the original toolset used in the first MSP simulation in the AOL in 2006 . An extensive suite of functions could be configured to effectively support traffic management coordinator (TMC), area supervisor (Sup), and MSP positions. New tools for situation assessment, planning and plan coordination were distributed throughout the ANSP operators to create a common understanding of the current and predicted situation in order to assess available options for the management, communication, and execution of proposed plans. Although the underlying technical infrastructure is described in Ref. 10, an overview is given here: all operator stations access an information management system for retrieving and providing information. Operators can use voice and data comm. to communicate between each other. Traffic flow planners use functions provided at their workstations to create provisional trajectories that can be coordinated with other traffic management coordinators, supervisors, or controllers. Provisional trajectories for single or multiple aircraft can be sent via the automation for review at other planning stations. Once the trajectories are ready to be issued they can be sent to the sector controllers for execution. Sector controllers evaluate if they pose a separation problem and send the trajectory changes to the aircraft as necessary. Under certain situations, planners can send downstream trajectory changes directly to the aircraft. Operationally the exact rules will have to be determined, but in the MSP simulations a

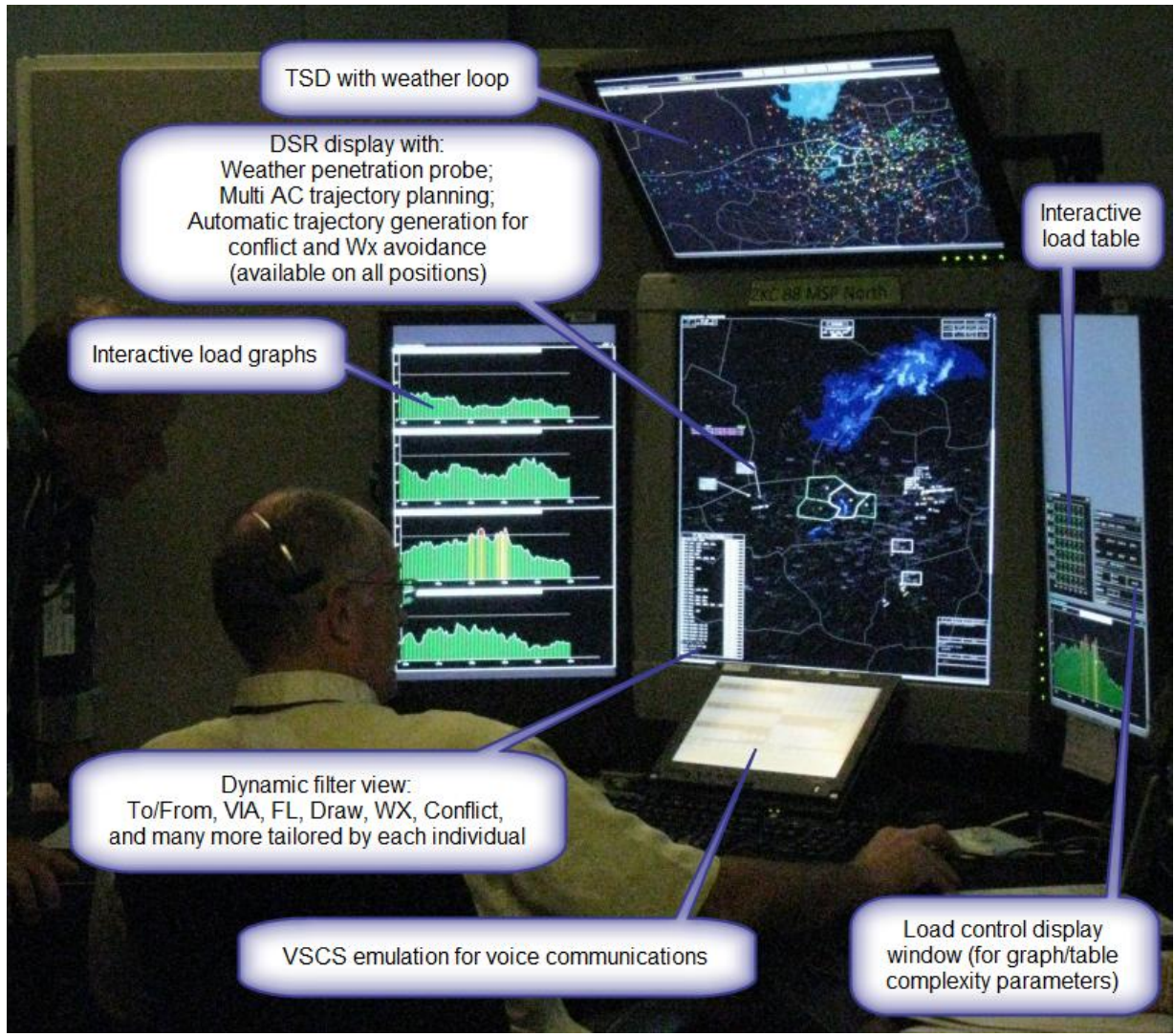

Figure 2. Multi sector planner during 2009 simulation.

American Institute of Aeronautics and Astronautics 
simplified rule allowed traffic flow planners to send trajectory changes to the aircraft if the first change point was at least 30 minutes away. Similar to the controller stations, the planning stations rely on accurate trajectory predictions to enable their functions.

Figure 2 shows a planning station and indicates some of the MSP capabilities. Real-time filtering and analysis tools provide for traffic flow, sector load and complexity assessment. Multi-aircraft trial planning functions provide options for previewing the impact for several trajectory changes on the overall situation. Any plans can be sent to other operators for their review. A short summary of the central new trajectory management functions follows.

\section{Traffic Flow Assessment}

In order to assess the traffic flow within a large congested airspace, new dynamic filter capabilities have been prototyped that allow operators to highlight specific subsets of aircraft. All traffic can be filtered such that only aircraft that fly to or from specific airports, or via designated routes, waypoints, or altitudes are highlighted. Aircraft can also be highlighted if their flight path passes through specific sectors, dynamically drawn objects, or forecasted convective weather areas. Filters can be combined, dynamically added, deleted or edited and color-coded. Aircraft that do not pass the filter test are pushed into the display's background; aircraft that meet the selected criteria are brought into the foreground. Figure 2 shows an example of how operators can use these filters. When the picture was taken, the operator was examining reroute options around a convective weather cell. Therefore, he selected a filter on the Display System Replacement (DSR) display that highlighted only aircraft that were predicted to penetrate the convective weather area within the next 30-90 minutes.

\section{Sector Load and Complexity Assessment}

Similar to traffic management tools today, traffic loads for sectors are computed as the number of aircraft predicted to be in the sector for a given time frame. The results are presented in tables and graphs. When the operator selects a specific time slice the aircraft that contribute to that specific load are also highlighted on the display. In order to account for complexity factors that go beyond the number of aircraft, the graphs and tables can be switched to show only subsets of the aircraft, such as the unequipped and transitioning aircraft, aircraft predicted to be in conflict, or aircraft predicted to penetrate weather hazards. In addition to these values a real-time estimate of the sector complexity is also computed. The complexity calculation includes the factors described above as well as the sector shape and size. Therefore, operators can use the complexity values instead of the total number of aircraft to have a more accurate estimate of the workload within any given sector. Results presented later in this paper indicate that planning controllers ranked this complexity computation among the highest rated overall tools.

All load graph and table values reflect active trajectories. Predictions for provisional trajectories are given whenever new trajectory plans are viewed. These plans could have been initiated at the station or received from other stations. Figure 3 shows an example of how the peak sector load impact can be previewed when planning two trajectory changes.

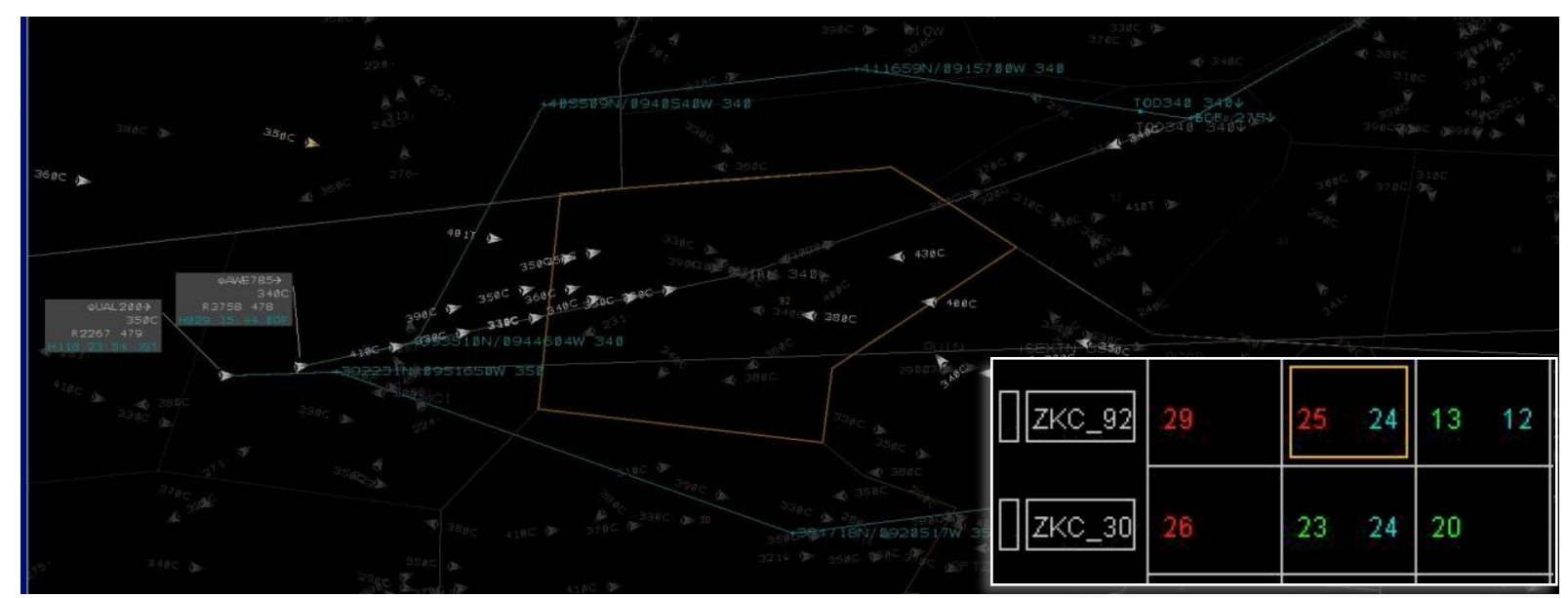

Figure 3. Two trajectory changes being performed via lateral trial planning. The load tables indicate the new peak aircraft counts in the impacted sectors (in cyan). 


\section{Multi Aircraft Trajectory Planning}

All the automation-assisted trajectory planning functions that exist at the tactical controller positions ${ }^{11}$ are also available at the planner positions. In order to assess the impact of providing reroutes for an entire flow of traffic, change altitudes on multiple aircraft, or conduct other flow based trajectory management tasks, the planner can create a selection of several aircraft and manipulate their trajectories at the same time. This multi aircraft trajectory planning can be done graphically and/or via keyboard entries. All trajectories can be probed for conflicts and hazard penetrations as desired. Figure 3 shows a trial plan that moves two aircraft around a busy sector. The load table indicates how the peak number of aircraft will change when these new trajectories are implemented.

\section{Plan Coordination}

Plans can be coordinated between traffic planner/manager stations for review. A single command can send a selection of trajectories to different stations. The receiving operators can review the plan using their own complexity assessment tools and approve or disapprove proposed trajectory changes. Once a plan has been agreed upon, it can be sent to the sector controller or directly to the aircraft, under certain conditions. Coordination with area supervisors should precede trajectory changes impacting operations in the area. Each individual trajectory can be reviewed by the sector controller. When acceptable he or she sends the trajectory change to the aircraft. An approval message is automatically returned to the originator of the trajectory change and a new trajectory amendment is made in the information management system.

\section{Method: Experimental Design of the MSP simulation in 2009}

The MSP study design is described in detail in Ref. 2. The following is a brief summary of this design as it explains why some of the results presented in Section IV are gathered from different operators.

\section{A. MSP Study Design}

As indicated before, a particular focus of the MSP simulation was to determine the need for creating a new staffed MSP position to perform MSP operations. To address this question, two test conditions with different ANSP team configurations were compared: a baseline "No-MSP" condition with TMCs, Sups, and radar controllers, against the "MSP" condition where MSPs were added to this baseline team configuration.

MSP tools were given to MSPs as well as TMCs and Sups in both conditions. MSPs and TMCs modified trajectories for flow purposes but at different look-ahead horizons (30-60 min for the MSPs and 45+ min for the TMCs). Sups used the tools primarily for situation assessment purposes. Traffic scenarios consisted of convective weather and traffic load problems involving over 1200 aircraft and spanning several facilities in the central United States.

The 'test' airspace included a 'high fidelity' area in the eastern half of the Kansas City Center (ZKC) that was staffed by most of the test participants: four air traffic controllers, one area supervisor, one TMC, and two MSPs, along with three retired controller 'confederates' (see Figure 1, green and orange sectors). The airspace surrounding these ZKC East test sectors was staffed primarily by confederates who managed combined sectors and areas and was managed by two more MSP test participants and a confederate TMC.

The experiment was conducted in the summer of 2009 in two separate two-week simulations. The results of the first simulation were used to improve the tools and procedures such that the second simulation represented a valid experimental environment.

\section{B. MSP Tools Data Collection}

The MSP tools data presented in this paper were collected from the MSP, TMC and Sup test participants of the second simulation. The MSPs were four operationally current air traffic operators who had experience as both area supervisors and traffic managers. The TMC and Sup were also both operationally current in their respective roles. The usage data presented subsequently were recorded by the simulation system (MACS) and later analyzed by the research staff. Subjective tools assessments were gathered after the simulation as part of a larger set of paper questionnaires that were administered on the last day of the simulation before the debriefing.

\section{Summary of Results}

This section contains high level summary of results regarding the provided toolset. Results regarding individual display elements and tools are presented in Section V. 


\section{A. Usefulness and Usability of Primary Planning Display Elements}

The primary planning display elements for conducting multi sector planning functions are described in Section II and depicted in Figure 2. These elements were:

- $\quad$ Traffic Situation Display (TSD)

- DSR Traffic view display

- $\quad$ Load Tables

- Load Graphs

- Dynamic Aircraft Filters (AC Filters)

The operators rated the main elements of the overall toolset in post simulation questionnaires on a scale of 1 (not at all useful/usable) to 6 (very useful/usable). The term "useful" was used to indicate the support that the tool/feature provided for the planners to complete a task, whereas the term "usable" was used to indicate the difficulty of actually performing an action with the tool/feature within the software. Figure 4 summarizes the results for the different operators.

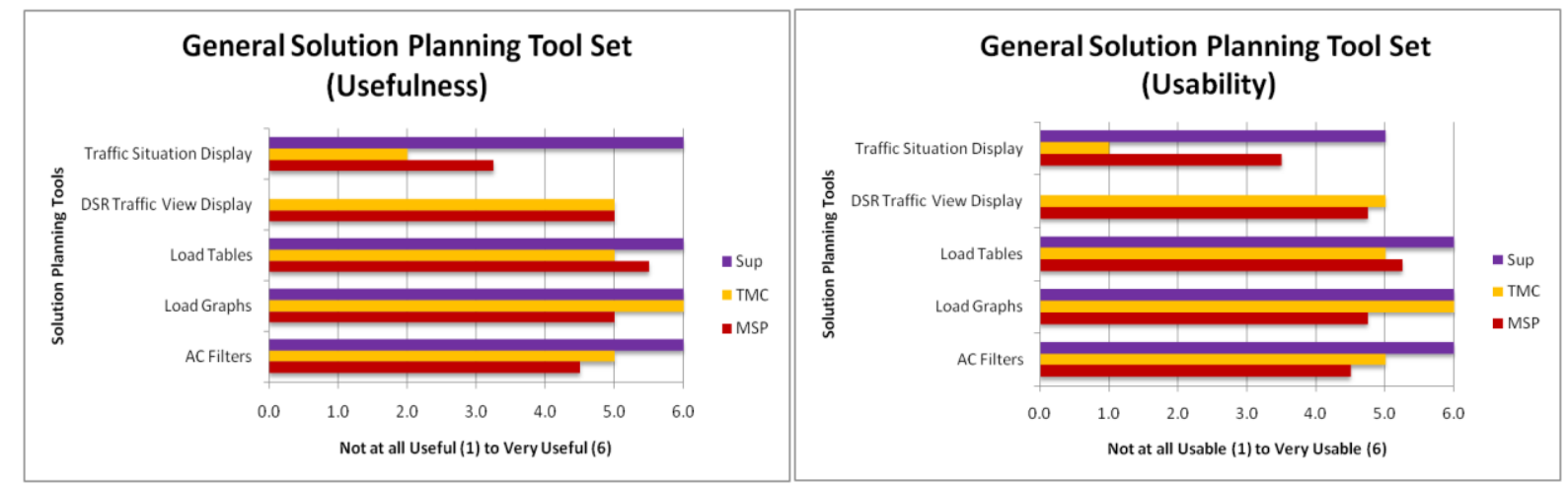

Figure 4. Overall toolset usefulness and usability ratings.

The TSD in this simulation did not have all the full capabilities it has in the field and was used more as a general overview of the traffic flows and the weather. MSPs and the TMC did not find the TSD very useful or usable for their planning purposes. The Sup rated the TSD very useful and usable but commented that it needed to function like the TSD in the field to be really usable. Their combined strategies for using the TSD included the color-coding of the directional traffic to help with Traffic Load problems and the current and future predicted weather loop to assist them in weather rerouting in Weather problems.

The DSR was rated as useful and usable for the TMC and MSPs. The Sup did not use the DSR so was not asked about it in the post-sim tools questionnaire. Again, the DSR was the primary interface used to view all the traffic and to perform the trial plan rerouting functions. The DSR was essential in conjunction with the load tables, load graphs, and the AC filter to determine if action was needed, and then to identify the appropriate solution. The biggest problem reported with the DSR was the inadvertent picking of underlying or nearby aircraft (target symbols) when trying to click on a route or interactive flight data block.

The Load Tables were rated both highly useful and usable. The planners felt that the Load Table controls were necessary to accomplish their task and that the information was displayed clearly and was fairly easy to interpret. Some would have liked a bit more training and hands on time to better understand the selections available. Overall, the Load Tables were integral to deciding how to help or plan for sector load mitigation and weather rerouting.

The Load Graphs were also rated high in usefulness and usability. The planning team thought they were an excellent and required tool for a MSP position. The graphs were used extensively in both the weather and traffic load problems. They reported that the graphs displayed very accurate and useful information and were key in being able to pinpoint which aircraft were an issue, and eliminated a lot of the guess work. The Load Graphs were regarded as a great reference on how long the peak periods would last.

The usability and usefulness of the AC Filters were also rated fairly high. The strategy for using the AC Filter varied between all the participants and was dependent on the scenario. The ability to filter the different aspects of the traffic was important to the planning process. The users liked to be able to make their own filters and it helped them identify the traffic flow patterns and build effective rerouting strategies. 


\section{B. Top Rated Tools}

All MSPs rated the usefulness and usability of a total of 68 functions on the planner workstations. The overall ratings were high with an average of 4.47. Figure 5 depicts the thirteen highest rated tools and functions on the MSP positions from the four MSP participants.

Air/Ground data link was rated as the most useful and usable tool. The remaining 11 tools and functions that follow were also rated very high at 5.75. Trial planning functions in general and route trial planning in particular were also part of the top ten. The Load Tables and Load Graphs were among the remaining highest rated toolsets. Also notable in this chart is the high rating of the complexity values' usefulness. Since using complexity instead of aircraft count was an important new feature, this particular item is discussed in some more detail in the next section.

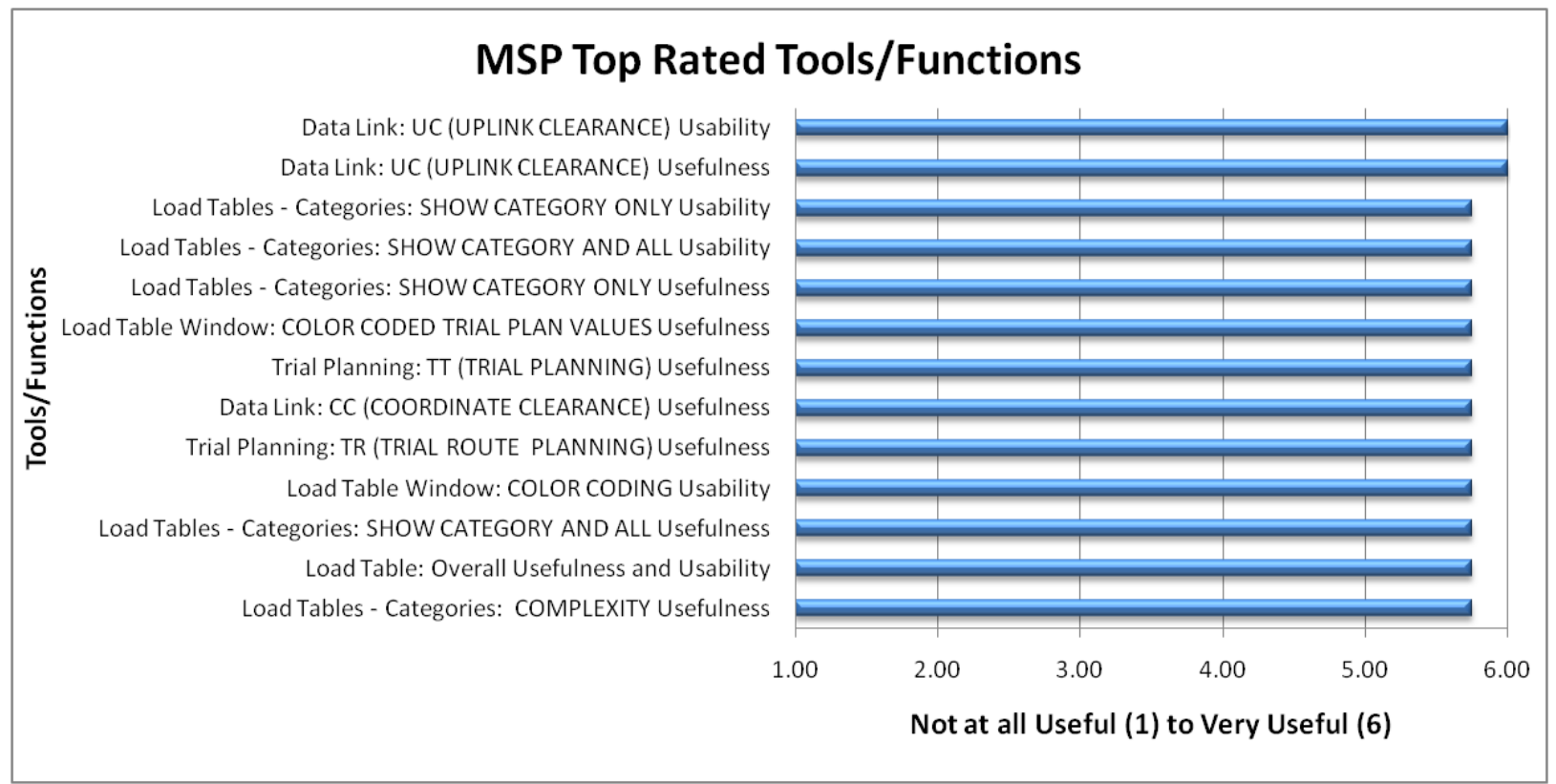

Figure 5. Top 13 MSP tools as rated by the MSPs.

\section{Complexity Value}

An important part of this simulation was to evaluate replacing or augmenting a purely traffic count based sector load assessment with complexity parameters. Therefore, a real-time complexity prediction value was computed in this simulation and made available to the MSPs, TMC, and Sup. The complexity prediction was in addition to the aircraft count prediction and various complexity contributors including weather penetration, traffic conflicts, and number of transitioning aircraft. The complexity value was normalized to the Monitor Alert Parameter (MAP) value, so that operators could use aircraft count and complexity values on the same scale. A complexity value that exceeded the MAP value could be interpreted as causing excessive work load. The subjective assessments shown above in Figure 5 indicate that the complexity value was rated very useful. The complexity setting was accessed among other prediction categories via designated load controls. The data in Figure 6 show the percentage of time that each category was used during the simulation.

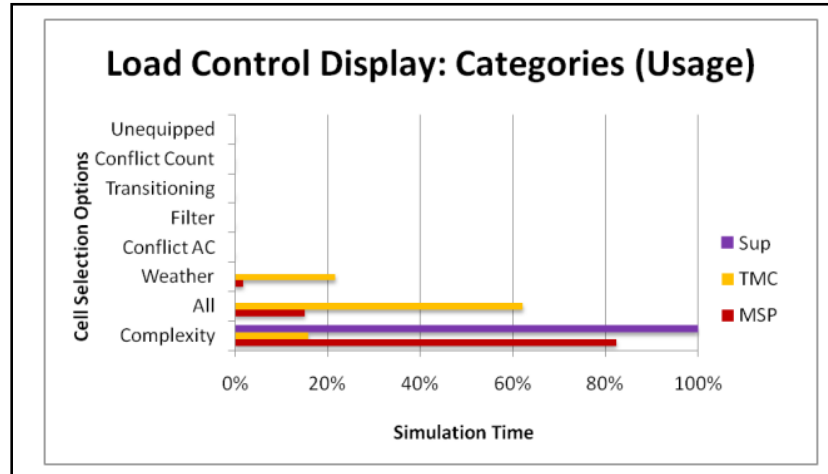

Figure 6. Usage of the complexity value.

The complexity value was very frequently used. The detailed results later in this paper indicate the complexity value was used most frequently in combination with aircraft count (see Figure 14 and Table 2). Individual factors 
contributing to complexity such as the unequipped and transitioning aircraft, aircraft predicted to be in conflict, or aircraft predicted to penetrate weather hazards were rarely selected.

\section{Detailed Results}

The results in this section are structured as follows. First, the tools for traffic flow assessment will be discussed, focusing on the Dynamic Aircraft Filters. This will be followed by the load and complexity assessment tools that include an interactive Load Control Display Window, Load Tables and Load Graphs with many new interactive features. This will be followed by the trajectory planning toolset. Results on tools for plan coordination are presented at the end of the results section. Additional tables containing details about some of the underlying mechanisms and additional descriptions and analysis can be found in the Appendix.

\section{A. Traffic Flow Assessment / Aircraft Filter}

\section{Description}

In order to assess the traffic flow situation within a large congested airspace, two displays were used. The Traffic Situation Display (TSD) indicated at the top of Figure 2 used color-coding for distinguishing specific flows and overlaid a weather loop that showed the history and projected future development of convective weather cells. The TSD was used primarily for general situation awareness and was not used interactively. Instead, an emulation of a basic current day DSR was enhanced substantially to serve as the primary interactive traffic display for the MSPs, TMCs and Sups (indicated in the center of Figure 2). These dynamic aircraft filter mechanisms were implemented for operators to easily highlight and select aircraft of interest. These filters could be defined and modified by the operators in real-time and allowed them to filter their primary traffic displays by various criteria including flows to

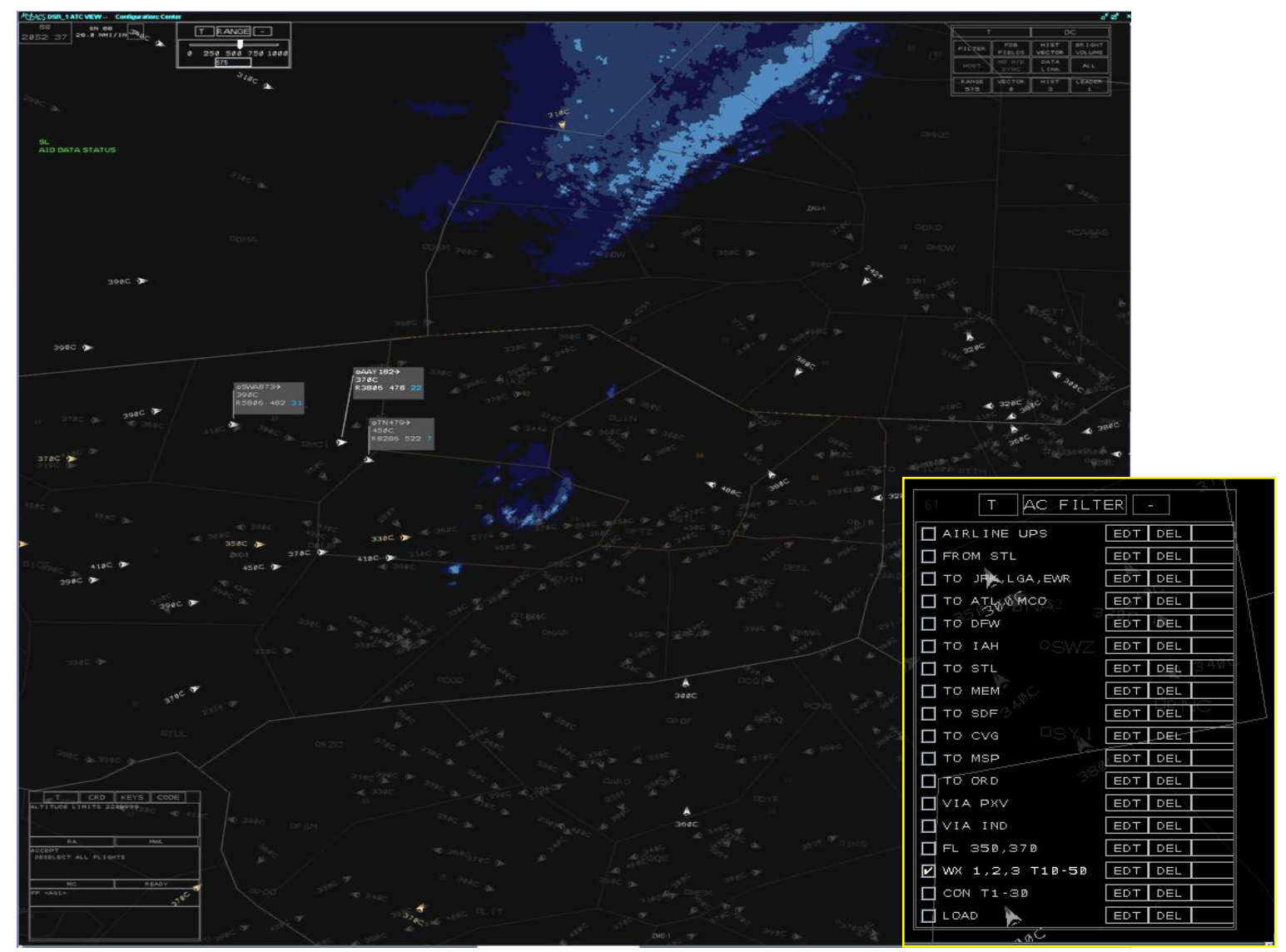

Figure 7. Example of DSR traffic display when aircraft are filtered by predicted weather penetration.

and from airports, predicted weather penetration, or cells selected on the Load Graphs and Tables. When filters were active, all aircraft that met the filter criteria were pulled into the displays foreground, while all other aircraft were pushed into the background. Figure 7 shows a DSR display and an AC Filter List in a situation in which the operator 
has selected to filter the traffic display such that all aircraft that are predicted to penetrate any of the three weather intensity levels (1 (low), 2 (medium), or 3 (high)) within ten to fifty minutes from now. The filter command for this filter was "WX 1,2,3 T10-50". An explanation of all filter commands is given in Table A1 in the Appendix. These aircraft are depicted with the bright chevrons, while the other aircraft are shown as grey background traffic. The operator has then selected three aircraft from this set that are shown with full data tags and a grey underlay. This multiple selection function is usually used in conjunction with trial planning and will be discussed later in this paper.

\section{Usefulness and Usability of the AC Filters}

The participant ratings depicted in Figure 8 show that filter categories such as the Weather Filter (5.5), Airport Filter (5.5), and overall usability and usefulness (5.5) were highly rated; however, relatively low ratings were received for several other categories such as Conflict Filter (2.75), Airline Filter (2.5), and the lowest rated Aircraft ID (1.75). The data suggests that specific filters are used often and rated highly useful and usable, while others provide little to no benefit for the given tasks in this specific environment. This supports the effectiveness of the approach of defining the necessary filters dynamically.

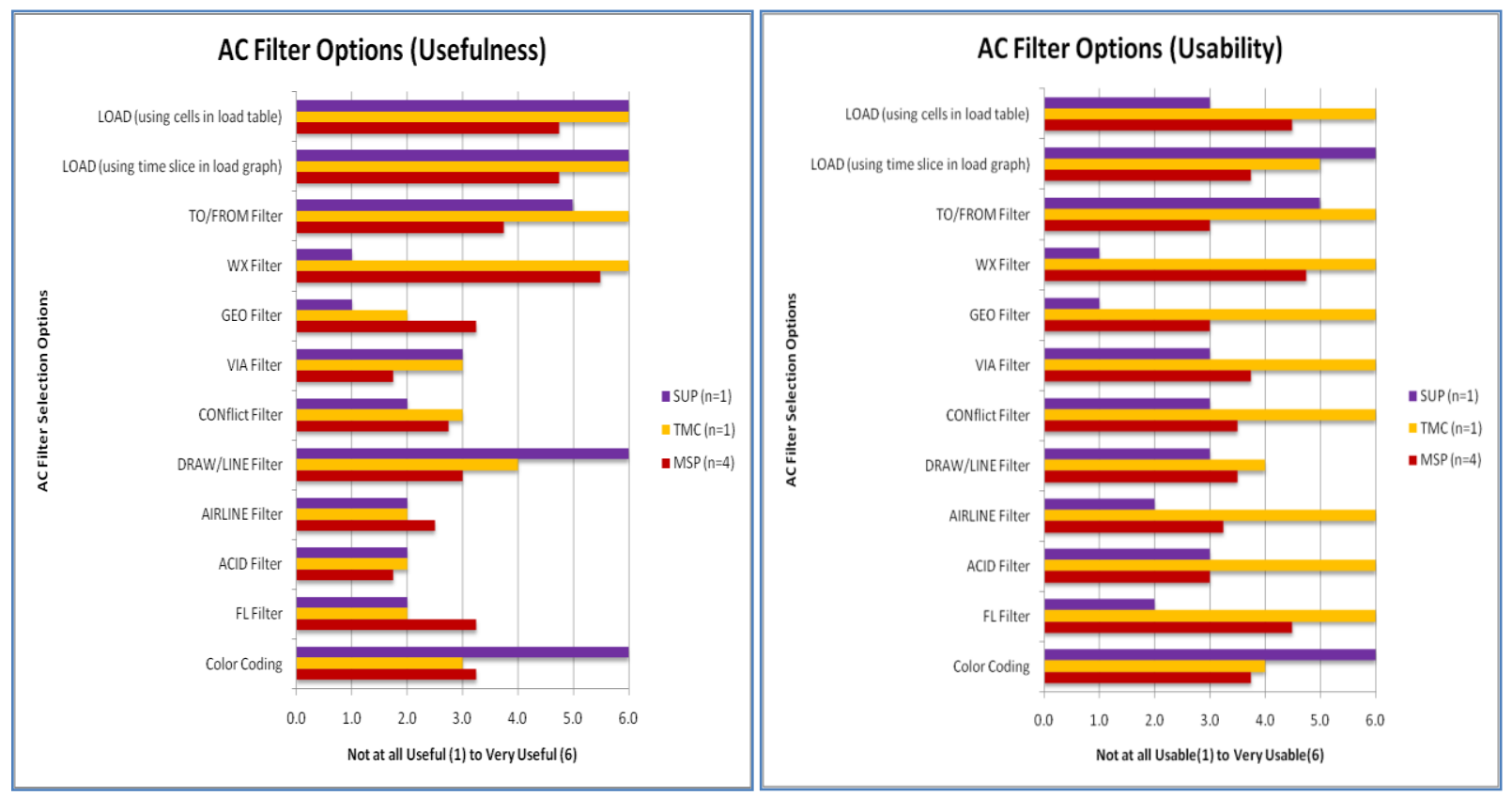

Figure 8. MSP AC Filter Usefulness and Usability ratings.

\section{Usage data of the AC Filters}

Usage of the AC Filters that allowed the MSPs, Sup, and TMC to filter their primary traffic displays by various criteria including flows to and from airports, weather penetration, cells selected on the Load Graphs and Tables, and others were analyzed. The data indicated the following usage distribution in the form of total counts (Table 1). Note that the description of the filter options is available in Appendix A.

Table 1. AC Filter usage counts.

\begin{tabular}{|l|c|c|c|}
\hline \multicolumn{1}{|c|}{ AC Filter Options } & MSP ( $\mathrm{n}=4 \times 8$ runs $)$ & TMC ( $\mathrm{n}=1 \times 16$ runs $)$ & SUP ( $\mathrm{n}=1 \times 16$ runs $)$ \\
\hline Selected LOAD & 159 & 96 & 66 \\
\hline Selected TO & 171 & 162 & 87 \\
\hline Selected FROM & 3 & 0 & 0 \\
\hline Selected WX & 80 & 13 & 0 \\
\hline Selected VIA & 0 & 1 & 0 \\
\hline Selected CON & 2 & 0 & 0 \\
\hline Selected LINE & 2 & 0 & 0 \\
\hline Selected GEO & 59 & 0 & \\
\hline
\end{tabular}

9

American Institute of Aeronautics and Astronautics 


\begin{tabular}{|l|c|c|c|}
\hline Selected AIRLINE & 5 & 0 & 0 \\
\hline Color Filter & 1 & 6 & 61 \\
\hline Create New Filter & 10 & 6 & 7 \\
\hline Deselect ALL & 12 & 78 & 1 \\
\hline
\end{tabular}

All participants used the LOAD filter and TO filter options the most. The LOAD filter was the main filter as it displayed traffic chosen directly from the Load Table and Load Graphs. The TO filter was especially useful because the planner could manipulate the traffic headed to the same airport during a specified length of time. The FROM, VIA, CON (conflict), LINE and AIRLINE filters were hardly ever used. The DRAW, AIRPORT, ACID and DIR filters were never used. The WX Filter was used more by the MSPs and TMC than the Sup. The ability to color-code the filter selections was primarily used by the Sup, whereas the MSPs and TMC did not use the color options.

\section{B. Load and Complexity Assessment}

The primary tools for assessing the traffic load consisted of the Load Display Control Window, the Load Table Window, and the Load Graph Window. All of the load tools were located to the side of the planner's DSR display and had interactive features. Figure 9 shows how these tools interacted. A supervisor selected a complexity peak in the load graph (highlighted in pink) and by doing this was able to view all aircraft that were included in this peak on the DSR traffic display (highlighted in green). So the supervisor could easily assess which aircraft would be in the sector of interest at the time the complexity peaked.

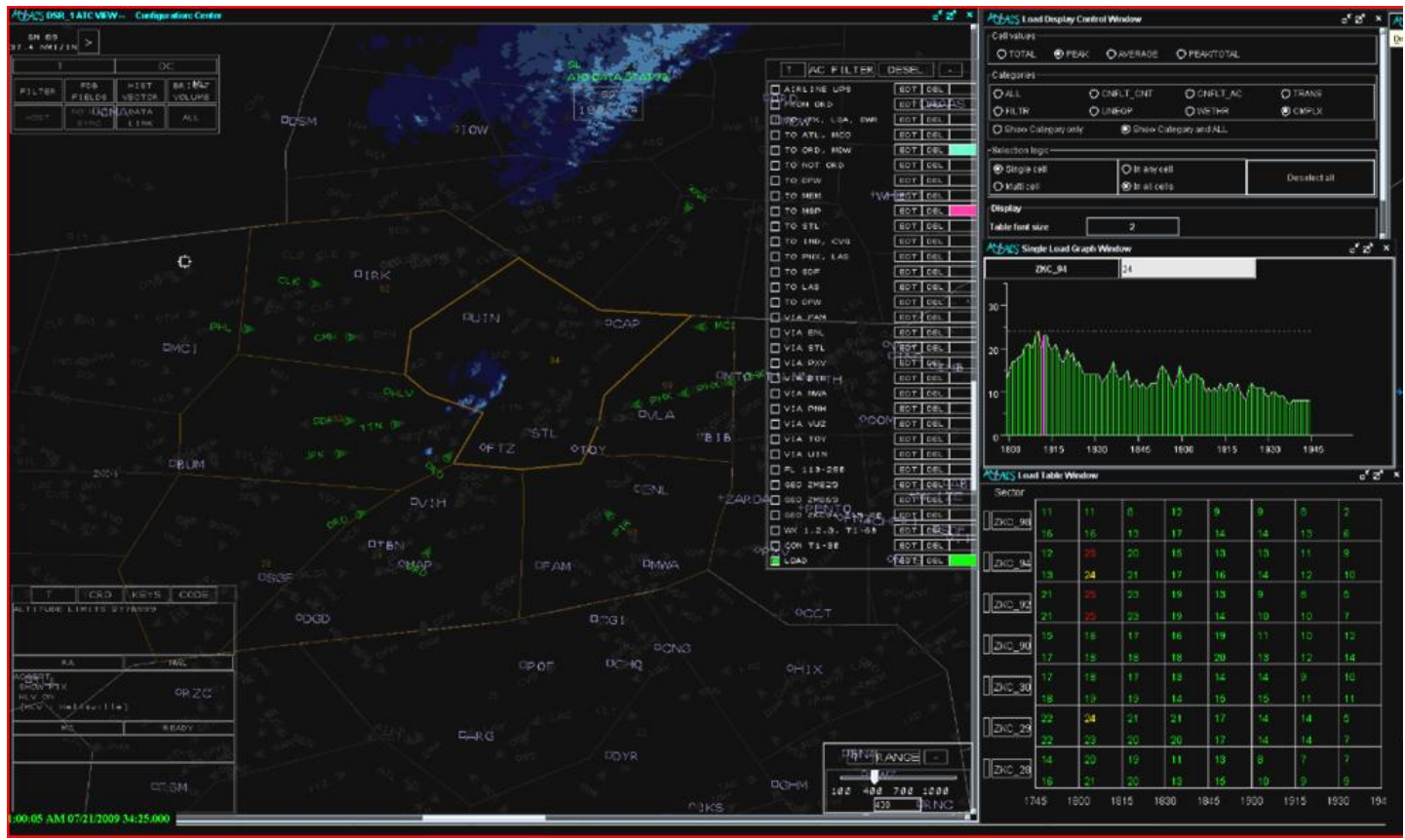

Figure 9. Load and Complexity Assessment Tools

\section{Description of the Load Control Display Window}

The Load Display Control Window was located on a side panel and enabled the user to select the data that were displayed in the Load Graphs and Load Tables. Furthermore, it drove the selection logic in the Load Table and Load Graphs (i.e. the interactive parts of the Load Table and Load Graphs were populated with values generated by the user selection in the Load Control Display Window). There were three main areas in the Load Control Table: Cell Values, Categories, and Selection Logic. Cell Values let the operator select whether to see the total, peak, average or a combination of peak and total traffic count for a given value in the Load Table. In the Categories area the operator could select which aircraft to include as a function of their characteristics. Additional selections could be made in the Selection Logic area on how more than one cell in the Load Tables should be combined. The details are in Appendix B. 


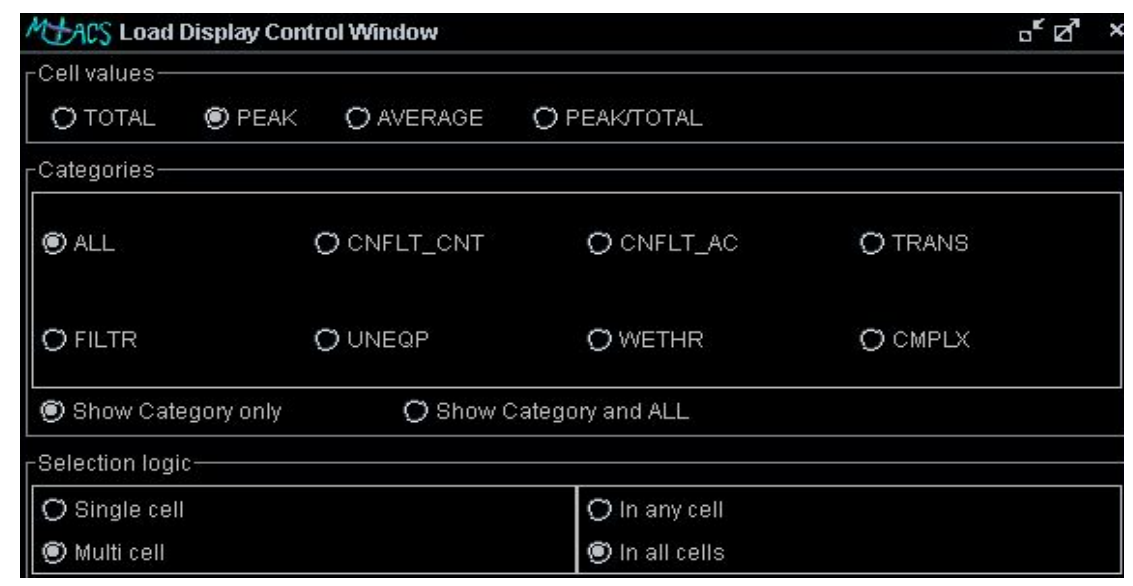

Figure 10. Load Display Control Window options.

2. Subjective Assessments of Load Control Display Window

The planning team rated Peak as the most useful and usable option of the four in the Cell Values area (Figure 11). Though each option was generally usable, the usefulness of Average and Total was on the low end; however, the Sup rated Total as very useful. Peak/Total received moderate ratings for usefulness. The actual usage data below supports that Peak Cell Values were highly valuable.

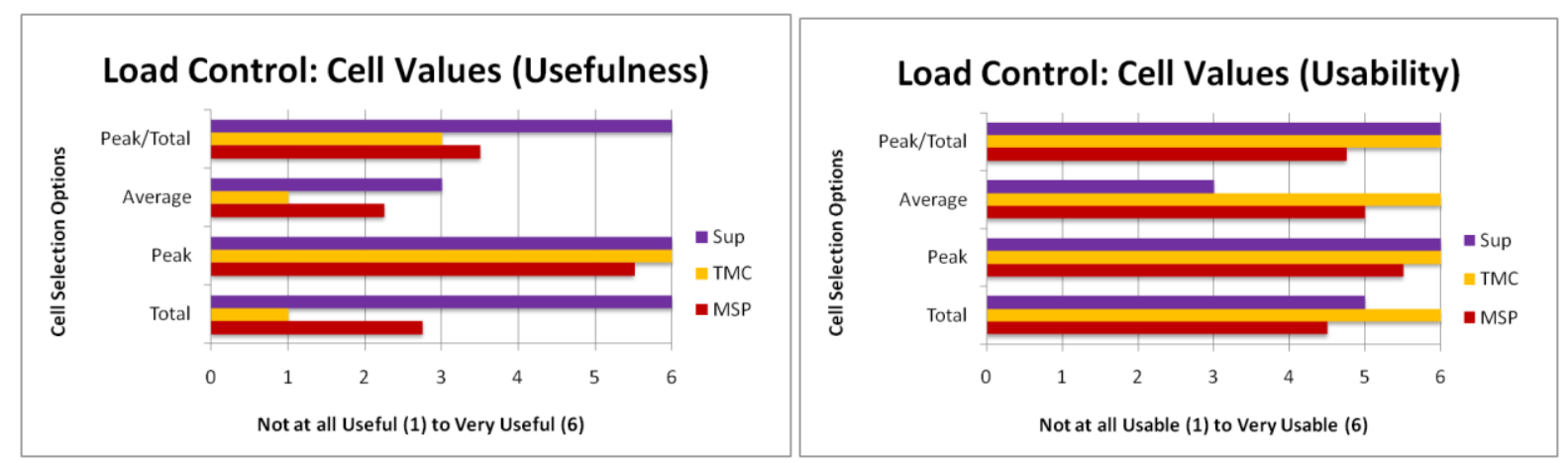

Figure 11. Cell Value usefulness and usability ratings.

The category that was considered most useful and usable by all three positions was All (Figure 12). Complexity, Show Category and All, and Show Category Only were also highly rated; however, the TMC gave moderate ratings for the usefulness of these categories. The Unequipped Category was not scored by all participants because it was not used in this simulation. MSPs and TMCs also found the weather category useful and the MSPs thought that transitioning aircraft and filtering by conflicts was useful as well. Even though these categories were specific contributors to complexity, the operators did not find them as useful and usable as the complexity value itself.

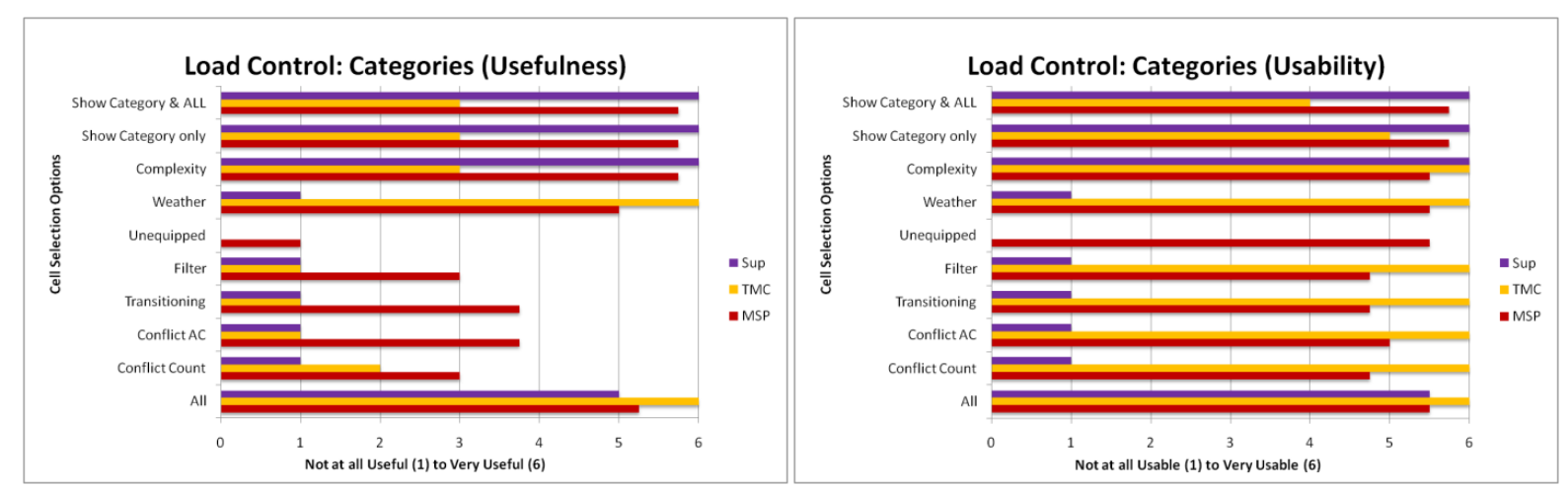

Figure 12. Categories usefulness and usability ratings. 


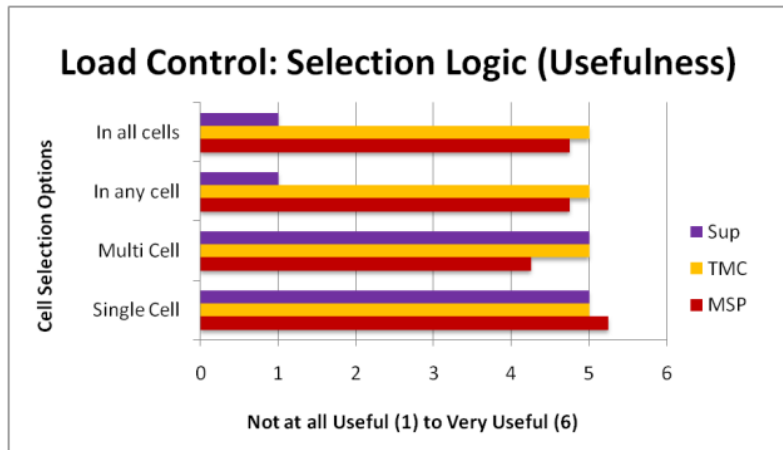

\section{Load Control: Selection Logic (Usability)}

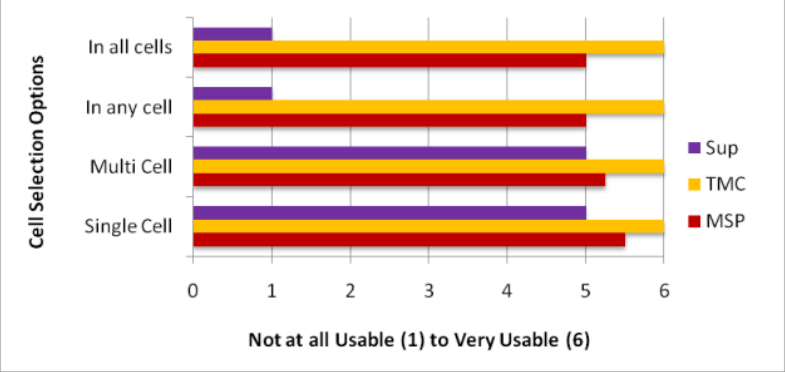

Figure 13. Selection Logic usefulness and usability ratings.

The Selection Logic options were generally rated as favorable (Figure 13); however, the Sup did not find the In All Cells or In Any Cell Selection Logic useful or usable. MSPs and TMCS found all different types of selection logic useful and usable. This is evidence that providing options for the operators to use the most appropriate selection logic for a given situation is appropriate.

\section{Usage of Load Control Display Window}

An important part of this simulation was to evaluate replacing or augmenting a purely traffic count based sector load assessment with complexity parameters. Therefore, the real-time complexity prediction value was computed in this simulation and made available to the MSPs, TMC, and Sup. The complexity prediction was in addition to the aircraft count prediction and various complexity contributors including weather penetration, traffic conflicts, and number of transitioning aircraft. The subjective assessments above indicate that the complexity value was rated very useful. The complexity setting was accessed among other prediction categories via designated load controls. The data in Figure 14 show the percentage of time that each category was used during the simulation. The figures show that the complexity value was very frequently used in combination with aircraft count (indicated as "ALL"), while individual factors contributing to complexity were rarely selected. This is in line with the subjective feedback above.

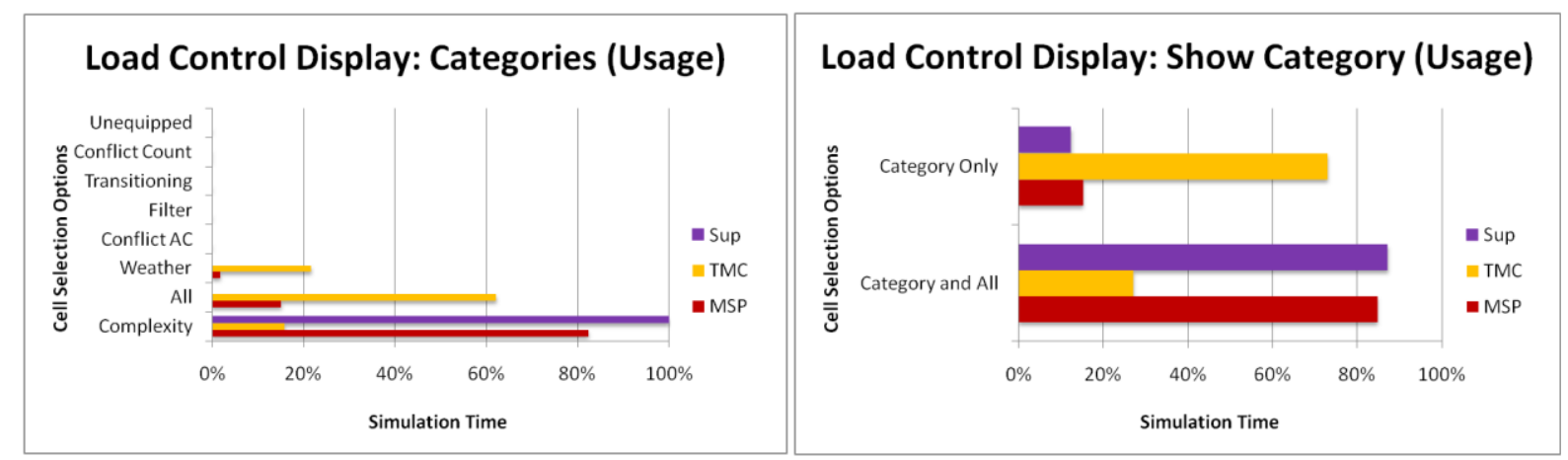

Figure 14. Usage data for Load display categories.

Further analysis of the usage data indicates the following usage distribution in form of percent time on each grouped selection. The items were grouped because no cell values were used independently. The four columns in Table 2 show the amount of simulation time spent using the specific configuration for each of the planning team positions and a combined overall tally. Only the top ten configurations are shown in Table 2 (i.e., the columns do not add up to $100 \%$ ).

Of the many possible configurations available to the planners, the MSP and Sup relied most heavily on "Peak", "Cmplx", "Show Category and All", "Single Cell", and "In Any Cell". The "Peak" traffic showed the highest peak number of traffic within the constraints of the 15-minute bins of the Load Table. The "Cmplx" selection displayed the value representing the overall complexity of the sector. "Show Category and All" displayed the "All" value (the value selected in Cell Value area) and whatever was selected in the Category area. "Single Cell" means only one cell at a time could be selected in the Load Table. "In Any Cell" would display all the aircraft that happen to be selected within the Load Table (e.g. there was only one cell selected in this case).

The TMC relied heavily on "Peak", "All", "Show Category Only", "Multi Cell", and "In All Cells". For this configuration, "Peak" remained the same; however, the following values were not. "All" displayed the number of all 
aircraft indicated in the Cell Value selection. "Show Category Only" displayed the Category selection but not the Cell Value selection. "Multi Cell" enabled the planners to select as many cells as they wished, and when combined with "In All Cells," more aircraft would be displayed on the DSR. The result of their inputs in the Load Display Control Window would be shown as values in the Load Table.

Table 2. Number of instances and percentage of use for grouped Load Control Display options.

\begin{tabular}{|l|l|l|l|l|}
\hline & \multicolumn{3}{l|}{ Percentage } \\
\hline & Overall & MSP & Sup & TMC \\
\hline peak, cmplx, all, single, all & $35.90 \%$ & $31.40 \%$ & $80.90 \%$ & \\
\hline peak, all, category, multi, all & $14.00 \%$ & $3.00 \%$ & & $50.00 \%$ \\
\hline peak, cmplx, all, multi, any & $11.20 \%$ & $21.40 \%$ & $0.10 \%$ & $1.90 \%$ \\
\hline peak, cmplx, all, single, any & $10.70 \%$ & $18.20 \%$ & $6.50 \%$ & \\
\hline peak, cmplx, all, multi, all & $5.70 \%$ & $6.70 \%$ & & $9.40 \%$ \\
\hline peak, cmplx, category, single, all & $3.90 \%$ & $1.50 \%$ & $12.50 \%$ & \\
\hline peak, all, category, single, any & $2.80 \%$ & $5.60 \%$ & & \\
\hline peak, wx, category, multi, all & $2.70 \%$ & & & $10.70 \%$ \\
\hline peak, wx, all, multi, all & $2.50 \%$ & $0.50 \%$ & & $8.80 \%$ \\
\hline peak, cmplx, category, multi, all & $2.10 \%$ & $1.90 \%$ & & $4.50 \%$ \\
\hline peak, all, all, multi, all & $2.00 \%$ & $0.60 \%$ & & $6.90 \%$ \\
\hline
\end{tabular}

\section{Load/Complexity Assessment: Load Table and Load Graphs}

The Load Table and Load Graphs were used by the MSPs, TMC, and Sup as a primary function that would instantly update various types of traffic load (e.g. weather, conflict, complexity) for a given sector at a specified time. Green values show air traffic below the specified Monitor Alert Parameter (MAP) value of 24, whereas yellow equaled the MAP and red exceeded the MAP.

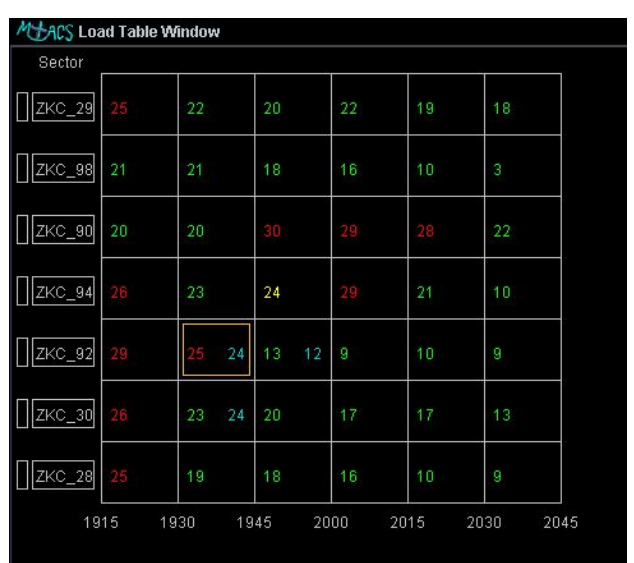

Figure 15. Load Table Window with sector ZKC_92 selected from 1930 to 1945.
The Load Table (Figure 15) was able to display more than one value at once for a 15-minute slice of time and could show a variety of traffic loads for several sectors. The Load Table Window is organized with the sectors owned on top and surrounding sector areas listed beneath them. Sector boundaries can be highlighted on the DSR by clicking on the small rectangle to the left of the Sector ID. Single Load Graph can be updated by clicking on the Sector ID box which replaced the previous graph. To select individual cell numbers for display on the DSR a highlighted magenta box appeared around the selected cell and the correct number of aircraft was highlighted on the DSR.

Load Graphs (Figure 16) were displayed for sectors that the participants needed and showed the level of traffic by the minute which could be selected to view future traffic volume on the scope. The Load Graph Windows were also located on the side panel area and displayed all the owned sectors predicted values from current to 1.5 hours into the future. The graphs were colorcoded to match the load window and displayed the data in 15 minutes increments and 30 second slices. The MAP value was set at 24 at the beginning of each trial but it could be moved as the user needed, though it was rarely ever moved. 


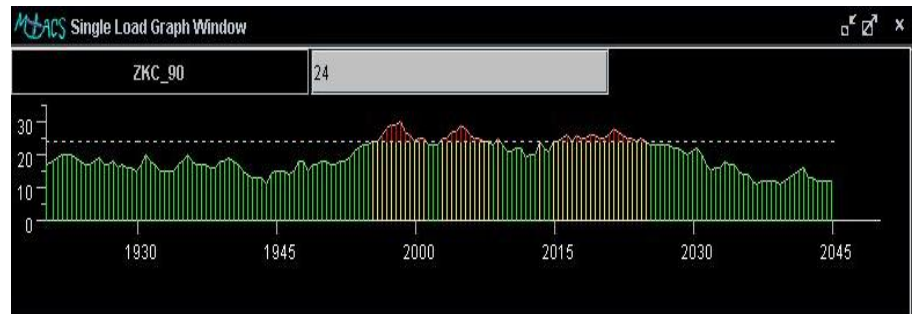

Figure 16. Single Load Graph Window with a selected MAP value of 24.

\section{Subjective Assessment of Load Table and Load Graphs}

Load Table subjective ratings were very favorable. The color-coded red, yellow, and green values were used to indicate above, on, or below the MAP value, respectively. Cyan numbers represented the future value of the cell if the planner implemented the trial plan that was currently displayed on the DSR. The numbers updated in real-time. Sector boundary highlighting would accent the chosen sectors from toggling a button to the left of the sector name. Cell selection was shown by a magenta box surrounding the values within the selected cells. The single sector graph selection displayed any of the sectors in the Load Table in graphical form in the Single Load Graph Window when selected by clicking on the sector name. Figure 17 shows that the Sup rated everything as useful and usable except for the color-coded trial plan values which were not meaningful because trial planning was not used by the Sup. The TMC also gave very high marks for each feature of the Load Table. The four MSPs' ratings were slightly lower but they still considered each feature of the table as highly useful and usable.

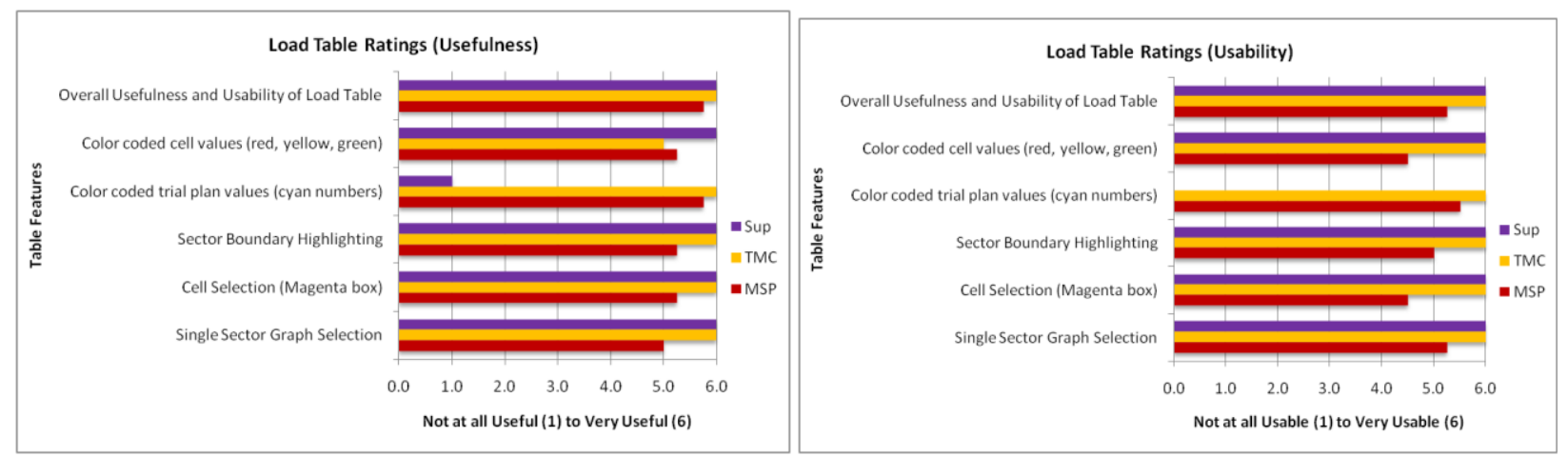

Figure 17. Usefulness and Usability of Load Table features.

The Load Graph subjective ratings data (Figure 18) were similar to the Load Table data. The color-coded red, yellow, and green bars were used to indicate above, on, or below the MAP value, respectively. Selecting specific time slices was possible by picking on the bars to display the current load on the DSR. Because it performed the same function as the Load Table cell selection, selecting a cell in the Load Table and a bar in the Load Graph were not possible at the same time, but rather used complementary as the Load Table's time slices were 15 minutes, whereas the Load Graph's time slices were 30seconds. The Sup and TMC rated the graph features as highly as possible (6.0), while the MSPs were slightly more critical overall (5.0). The MSP rating for Selectable Time Slice (4.5) for both Usefulness and Usability show that it may benefit from additional improvements. 


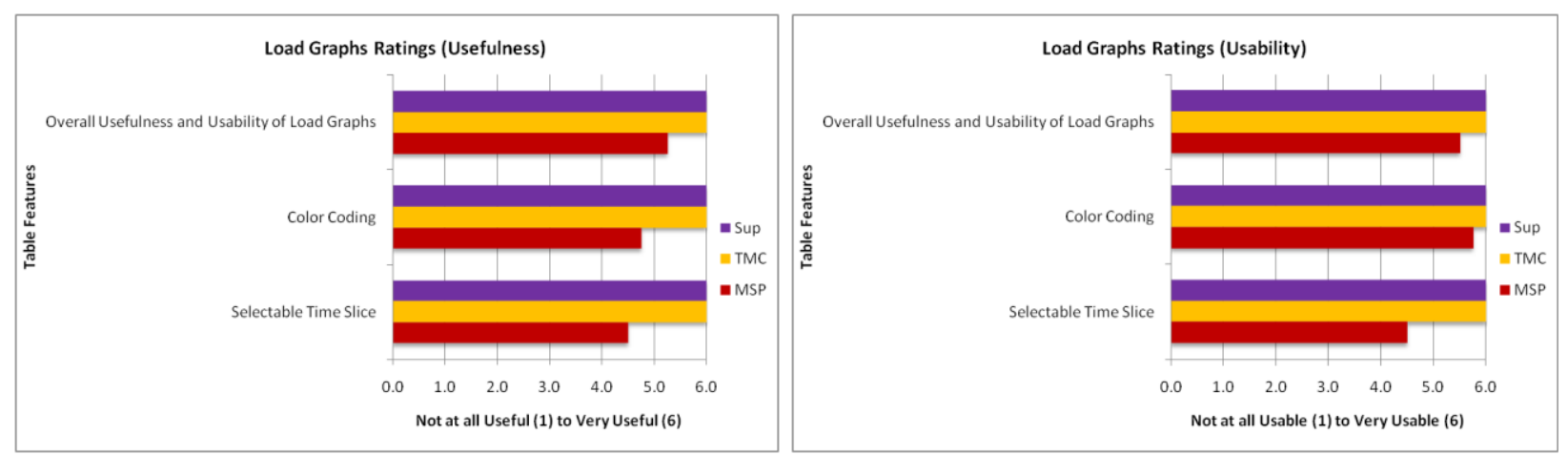

Figure 18. Usefulness and Usability of Load Graph features.

\section{Usage of Load Tables and Load Graphs}

While the Load Graphs and Load Table were permanently available for situation assessment, the planning team used the interactive features of the Load Table (63.8\%) more often than the Load Graph (36.2\%) to highlight the aircraft on the DSR traffic display. This result is probably biased by an artifact of the software used during the simulation that caused the slice selection in the Load Graph to be less stable than the cell selection in the Load Table. Figure 19 shows that the Load Table was used slightly more in the Weather condition than in the Traffic Load condition. The Load Graph was used less in the Weather condition than in the Traffic Load condition.

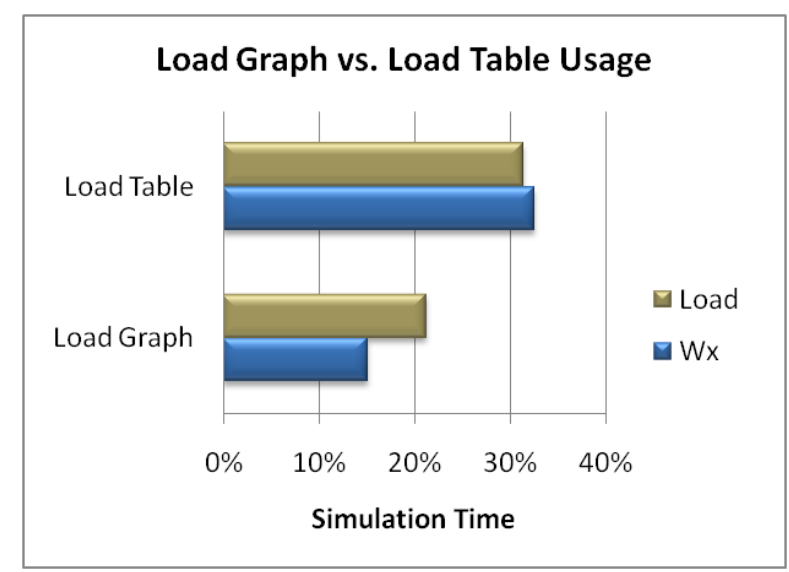

Figure 19. Load Graph vs. Load Table usage split by WX/Load conditions

\section{Trajectory Planning Tools \\ 1. Description}

This section describes the graphic trajectory planning tools that allowed the participants to develop and analyze trajectory based solutions to traffic load and complexity problems. When the traffic and weather situations created excessive complexity in certain airspace areas, the planners along with the Sup and TMC needed to be able to develop new plans. The primary method in the simulation was trial (trajectory) planning. As a new feature, a method for selecting multiple aircraft was implemented that allowed the participant to trial plan multiple trajectories simultaneously and assess their impact on the predicted sector complexity and load. This feature allowed operators to assess the impact of conducting coordinated flow manipulations, such as changing the altitude for groups of aircraft, or rerouting entire flows via new fixes. When multiple trajectories for multiple aircraft were overlaid on the screen the operator could also drag all trajectory lines simultaneously to create a common route.

There were a variety of ways to enter trial plan mode. The user could choose one or more aircraft to build a trial plan with and type commands into the DSR or use the interactive flight data block (FDB) trial plan features. The typed commands included FF (selected aircraft for group trial plan), TT (opened basic route trial plan), TA (choose specific altitude to trial plan), and TR (choose specific route to trial plan). The interactive FDB portals required 
picking on specific portions of the FDB which included the arrow next to the aircraft call sign (opened basic route trial plan and performed the same function as the TT command), magenta arrow to review a suggested trial plan from another planner or to review a trial plan already sent to others, the altitude portion of the FDB started an altitude trial plan, and the weather or conflict number to start a trial plan to solve for the conflict or the weather penetration. A description of each trial planning method can be found in Appendix C.

\section{Subjective Assessment of Trajectory Planning Tools}

Figure 20 shows the usefulness and usability ratings of the TMC and the MSPs for the four major trial plan methods which were generally considered highly useful and moderately-to-highly usable. The lower usability rating seemed to be primarily related to difficulties in selecting the appropriate display features and the sometimes slow responsiveness in the MSP station displays. The performance of the MSP station displays was impacted by running commercial screen recording software to record the screen contents and operator inputs. The associated slow down in responsiveness impacted the usability of interactive graphical tools. This underlines the need for highly responsive displays to be able to use these graphical and computationally intensive tools effectively.

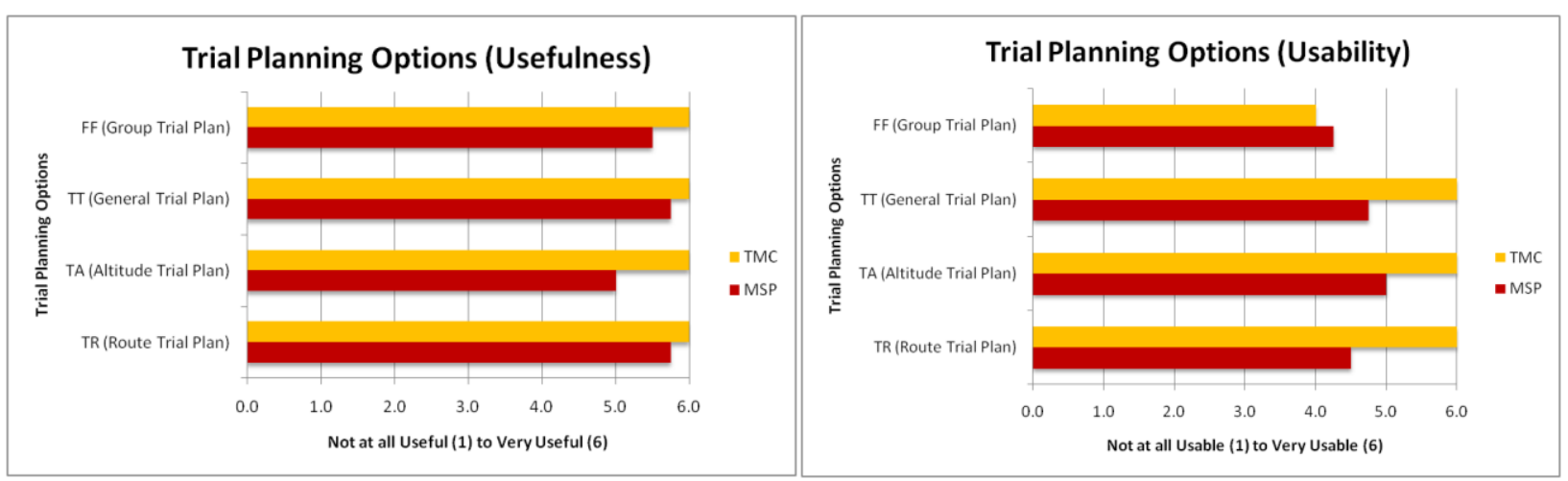

Figure 20. Usefulness and Usability of Trial Planning Options.

\section{Usage of Trajectory Planning Tools}

Figure 21 shows the percent of actual trial plan usage broken down by the various trial plan usage options for the four MSPs and the TMC over all runs. The new multi-aircraft trial plan feature (FF) was the most used method of opening a trial plan. This method allowed the planners to drag a box around several aircraft or picked individually to select all of the aircraft for a given trial plan.

The FF command was used for $34 \%$ of all trial plans, or 201 times, encompassing 980 aircraft. Typing TT $(30 \%, 883$ times) was the second most used way of opening a trial plan. The third most used method of opening a trial plan was opening the trial plans sent as coordination plans (CP) by the other planning positions. Clicking on a Pink Arrow in the Flight Data Block (FDB) (10\%, 298 times) would allow the user to look at other trial plans being sent to them for coordination purposes. Typing TA (9\%, 268 times), typing TR $(6 \%, 175$ times), and clicking on the FDB trial plan portal arrow $(6 \%, 164$ times) rounded out the other most used methods for opening a trial plan. 


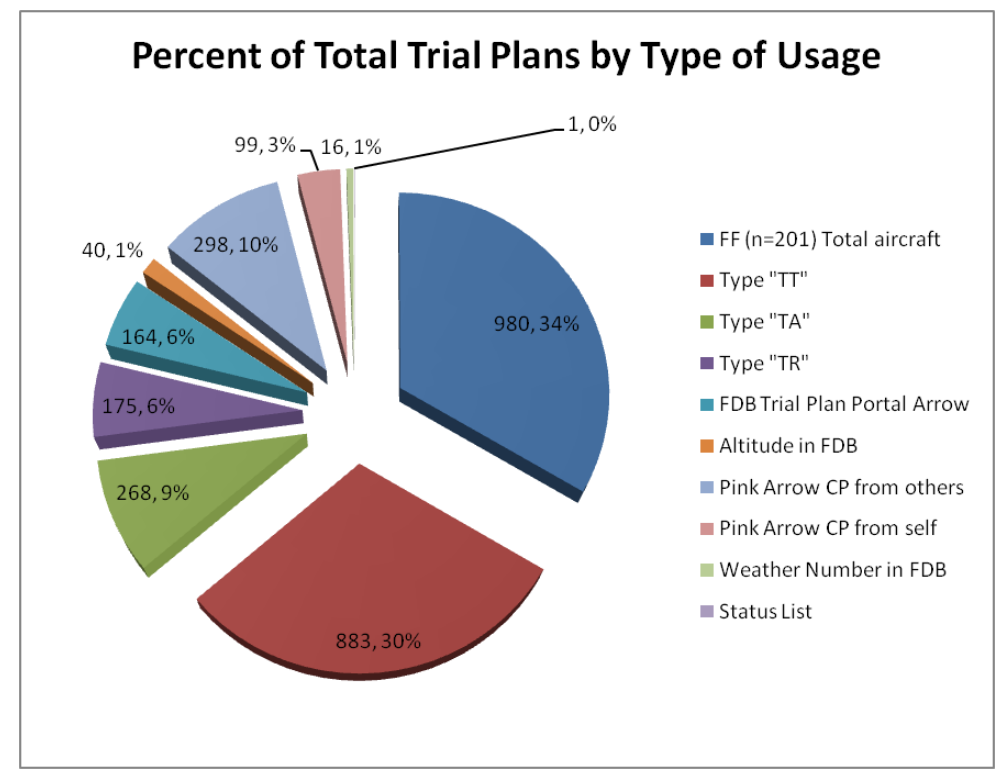

Figure 21. Percent of Total Trial Plans broken down by Trial Plan types.

Since FF was the most used method of trial planning and there were various ways to use the FF feature it should be explained in a little more detail. The planners averaged 4.9 aircraft per FF with the minimum number of aircraft being 2 and the maximum number of aircraft at one time was 52. The mode was 2 aircraft per FF. When an FF was started, one of the other methods of trial planning was necessary to perform the actual trial plan. An FF could be followed with a TT, TA or TR command. Figure 22 shows the number of trial plans with each FF method.

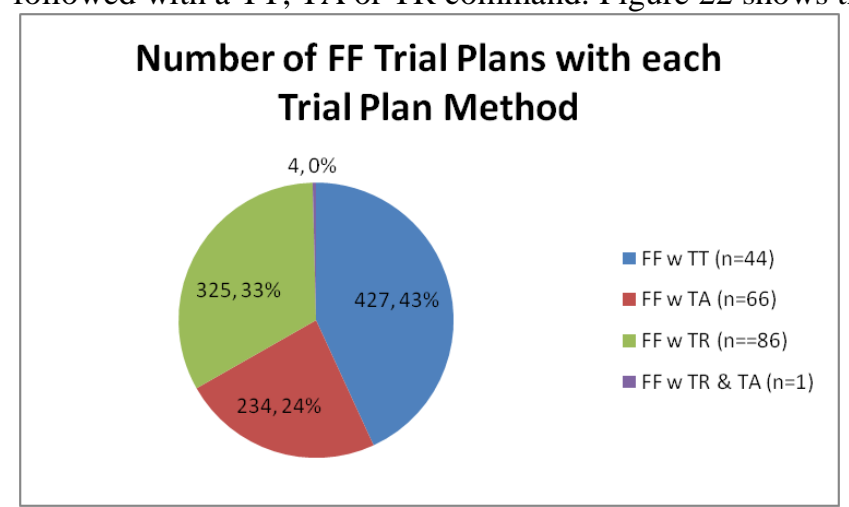

Figure 22. Number of FF Trial Plans using each Trial Plan Method

\section{E. Communication and Coordination \\ 1. Description}

Two forms of communication, data and verbal, were used for ground-ground and air-ground communications during the simulation. All verbal communications between MSPs, TMCs and Sups were conducted over a multichannel digital voice communication system, with no direct "face-to-face" verbal communications. Data comm was available to support planning of trajectory and flow modifications using " $\mathrm{CP}$ " (coordinate plan) messages. The message would be received at the intended station where the planner was able to "CY" (clearance-yes/accept) or "CN" (clearance-no/reject) the message, which was relayed back to original planner. MSP or TMC clearance requests could also be sent directly to the controller using a "CC" (coordinate clearance) message format that the controller could forward directly to the aircraft with the "UC" (uplink clearance) command. Alternatively, the clearance request could be indirectly relayed verbally by the area supervisor.

\section{Subjective Assessments}

Figure 23 details the planner team ratings on usefulness and usability regarding the means of communication. The communication mechanisms were rated mostly positive for both voice and data. Suggestions for improving 
particularly the exchange and bookkeeping of plans between planner positions were made. The communication of trajectories to the tactical controller positions was rated mostly useful and usable from the planner positions, while the sector controllers commented that some additional explanations and annotations would improve the communication path.

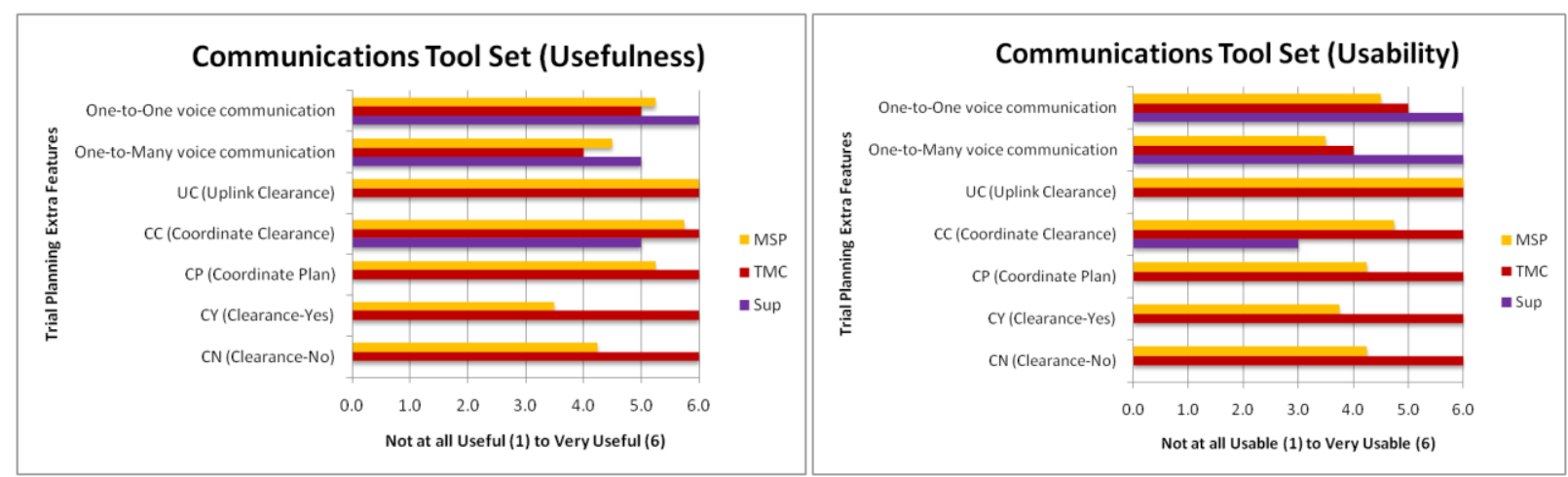

Figure 23. Usefulness and Usability of Communications Toolset.

\section{Requested Future Features}

The planning team commented that the TSD needed to have a way to color-code and label the different airports, display the proposed departures, and include the flow evaluation area and flow constrained area (FCA/FEA) tools. They all liked the weather prediction loop, but felt that it needed a time clock next to the prediction so that they knew what time frame they were looking at.

A requested feature for the DSR was a "See All" feature for their respective sectors to confirm their actions as MSPs. They also wanted a way to log or list the aircraft and/or the plans they developed for later reference.

The most requested Load Table feature was a cell value showing proposed departure aircraft, which was not feature incorporated into MACS. And in some cases, they wanted the ability to separate over-flights, arrivals, and departures within the cells. Another feature requested was the ability to look at the traffic load values of adjacent airspace ( 300 miles) rather than sectors and airspace predefined for them as they were in this study. Also, the TMC and some of the MSPs suggested the ability to show on the Load Table when an action has been taken to fix a high load number (e.g., a green bar through the red number). This bookkeeping action could have been taken by the TMC or by one of the MSPs. The indicator that an action had been taken would have saved time, eliminated multiple trial plans on the same aircraft, and eased the communication required to coordinate the actions.

Similar to their comments on the Load Table, the planning team felt the Load Graphs could have benefitted from displaying the proposed departures and how they might impact the sector(s). Another suggestion was to be able to select multiple slices of the graph peaks for examination. Some of the planners would have liked to see (e.g., "Quick look") the graphs for adjacent sectors as well as those in surrounding centers.

The planners thought it would be nice to be able to custom sort the options in the AC Filter . Another suggestion was to make the LOAD filter its own button, possibly next to the "Deselect All" button at the top. The DRAW and LINE filters were found to be beneficial, but difficult to use. The planning team wanted a new way of being able to define an area. The WX filter was nice to show aircraft that might need a reroute around weather, but it would have been nice to be able to specify a certain area or a single weather cell, rather than all weather cells.

\section{Conclusions}

An extensive suite of new tools and functions for multi sector planning for trajectory-based operations was implemented and evaluated. Overall, the usefulness and usability ratings of the MSP toolset broken down into 68 functions were high with an average of 4.47 on a scale of 1 (not very useful/usable) to 6 (very useful/usable). Air/Ground data link was rated as the most useful and usable tool while eleven more tools were also rated very high at 5.75. This includes trial planning functions in general and route trial planning in particular. Load Tables and Load Graphs and the usability of the complexity value were also among the top rated tools. 
The complexity estimate was among the highest rated tools. Particularly in the Weather problems, complexity was used as the primary measure instead of and in addition to aircraft count. Usage data and subjective ratings show that the single value complexity estimate was used far more than any of the contributing factors. Therefore a complexity value normalized to the MAP value proved to be a suitable concept for the simulated mid-term operations. Dynamic Aircraft Filters enabled operators to assess the situation from many different aspects. These flexible dynamic filters were preferred to the limited set of fixed categories available through the Load Table Controls.

Multi aircraft trial planning and selection was also found to be an effective and necessary means to create new plans that the planner benefited from.

While the general mechanisms of the simulated toolset appeared to be working well, few areas were identified for improvement. These areas include performance enhancements to improve the responsiveness of the tools and the inclusion of new features that would further increase the effectiveness of the overall toolset.

The toolset was rated very useful and usable overall. Many elements received high scores by the operators and were used frequently and successfully. Other functions were not used at all, but various requests for new functions and capabilities were received that could be added to the toolset.

\section{Appendix}

\section{A. Dynamic Filter Options}

Table A1: AC Filter Options

\begin{tabular}{|c|c|c|}
\hline $\begin{array}{ll}\text { AC } & \text { Filter } \\
\text { Option } & \\
\end{array}$ & $\begin{array}{l}\text { CRD/Keyboard Filter Command } \\
\text { FC }\end{array}$ & Filter Definition \\
\hline TO & FC TO [airport] or [ARTCC] & Filter aircraft to specific arrival airport(s) \\
\hline FROM & FC FROM [airport] or [ARTCC] & Filter aircraft coming from a specific airport(s) \\
\hline VIA & FC VIA [fix] & Filter aircraft going via a certain waypoint/fix \\
\hline FL (ALT.) & FC FL [alt] [alt] & Filter aircraft by altitude(s) \\
\hline $\begin{array}{l}\text { GEO } \\
\text { (SECTOR) }\end{array}$ & FC GEO [ZKC90] or [ZME] [T] & $\begin{array}{l}\text { Filter aircraft by sector ownership or ARTCC @ Time } \\
\text { X }\end{array}$ \\
\hline $\begin{array}{l}\text { DRAW or } \\
\text { LINE }\end{array}$ & $\begin{array}{l}\text { FC DRAW or LINE }[\mathrm{F} 1] \\
{[\mathrm{T} 15=35]}\end{array}$ & $\begin{array}{l}\text { Filter aircraft that will enter any "Draw Tool" defined } \\
\text { area @ Time X }\end{array}$ \\
\hline WX 1, 2, 3 & FC WX 1,2,3 [T25-45] & $\begin{array}{l}\text { Filter aircraft that are predicted to go into weather low } \\
\text { (1), medium (2), and high (3) @ Time X }\end{array}$ \\
\hline CONFLICT & $\mathrm{FC} \mathrm{CON} \mathrm{T1=30}$ & $\begin{array}{l}\text { Filter aircraft that are predicted to be in conflict at } \\
\text { Time } X(\mathrm{~T} 1-30 \text {, between now and } 30 \text { minutes })\end{array}$ \\
\hline ACID & FC ID [NWA123] & Filter aircraft by ID (NWA123) \\
\hline AIRLINE & FC AIRLINE [SWA] & Filter aircraft by airline (SWA) \\
\hline AIRPORT & FC AIRPORT [DFW] & Filter aircraft to/from this airport \\
\hline DIR & FC DIR [Heading Range 045-090] & Filters aircraft heading in a specific direction \\
\hline LOAD & FC LOAD & $\begin{array}{l}\text { Filter aircraft based on Load Table/Graph criteria } \\
\text { selected }\end{array}$ \\
\hline
\end{tabular}




\section{B. Load Display Control Options}

Table A2. Definitions of Options in Load Display Control.

\begin{tabular}{|c|c|}
\hline Option & Definition \\
\hline \multicolumn{2}{|r|}{ Cell Values } \\
\hline TOTAL & Total number of unique aircraft \\
\hline PEAK & Highest peak number of aircraft \\
\hline AVERAGE & Average number of aircraft \\
\hline PEAK/TOTAL & Show both highest peak \# of aircraft and total \# of aircraft \\
\hline \multicolumn{2}{|r|}{ Categories } \\
\hline ALL & Number of all aircraft indicated in Cell Value selection \\
\hline CNFLT_CNT & Total number of predicted conflicts \\
\hline CNFLT_AC & Total number of aircraft predicted to be in conflict \\
\hline TRANS & Number of aircraft transitioning (climbing or descending) \\
\hline FILTR & Number of aircraft that meet the AC filter criteria setting on DSR \\
\hline UNEQP & Number of unequipped aircraft \\
\hline WETHR & Number of Aircraft predicted to go through weather \\
\hline CMPLX & A number value representing complexity of sector workload \\
\hline Show Category Only & Shows the number in the Load Table of the category selected \\
\hline Show Category and ALL & $\begin{array}{l}\text { Shows the number in the Load Table of the category selected as well as } \\
\text { the ALL (value selected in cell value box) }\end{array}$ \\
\hline \multicolumn{2}{|r|}{ Selection Logic } \\
\hline Single cell & One cell selection in the Load Table \\
\hline Multi cell & Multiple cell selection possible in the Load Table \\
\hline In any cell & $\begin{array}{l}\text { "More" function, shows on DSR display aircraft that would fall into in } \\
\text { any cells selected on the Load Table }\end{array}$ \\
\hline In all cells & $\begin{array}{l}\text { "Less" function, shows on DSR display aircraft that would fall into each } \\
\text { and every cells selected on the Load Table }\end{array}$ \\
\hline
\end{tabular}




\section{Methods for Trial Planning}

1. FF (select aircraft for group trial planning)

The FF feature allowed the user to preselect more than one aircraft to start a group trial plan. Multiple aircraft were selected at once by dragging a rectangle around the chosen aircraft. Alternatively, the user could also pick individual aircraft to add or remove from the group. Selected aircraft datablocks were highlighted to indicate the selection (Fig. A1). The trial plan that followed was a route, altitude, or combined route and altitude solution.
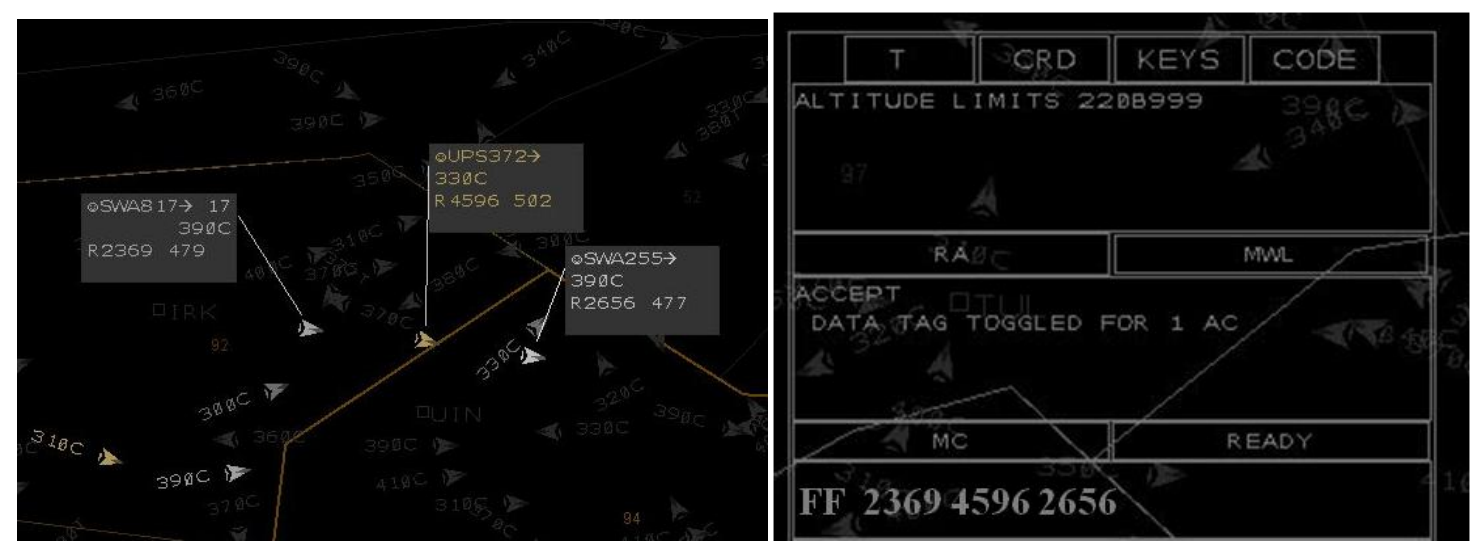

Figure A1. "FF" selected a group of aircraft for trial planning.

\section{TT (start interactive route trial plan)}

The TT command allowed the user to start a generic route trial plan. The current route of flight was drawn in the cyan trial planning color along with a drop down list of fixes along that route (Fig. A2). The planner could select new fixes in the list or drag the trial plan line with the trackball to manually create a dynamic flight path.

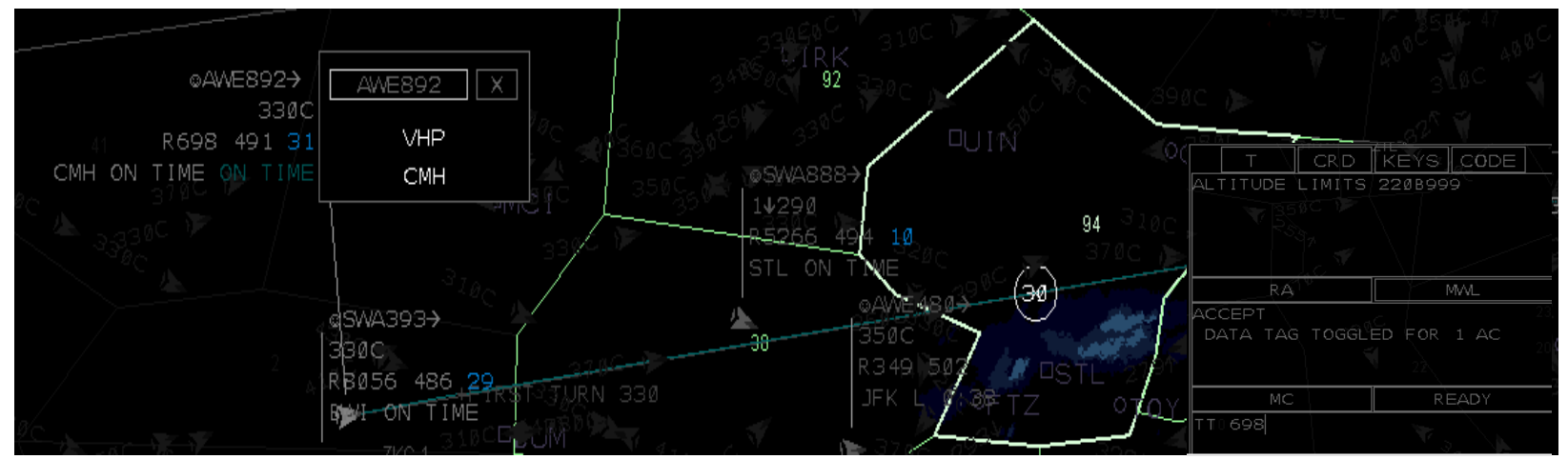

Figure A2. "TT" command presented the user with a trial plan.

\section{TA (altitude specific trial planning)}

The TA command allowed the user to start a specific altitude trial plan. The planner would pick on the altitude in the FDB to select a new altitude in a list, or they would type the TA command followed by the altitude and ACID to make appear the trial plan for that aircraft (Fig. A3). 


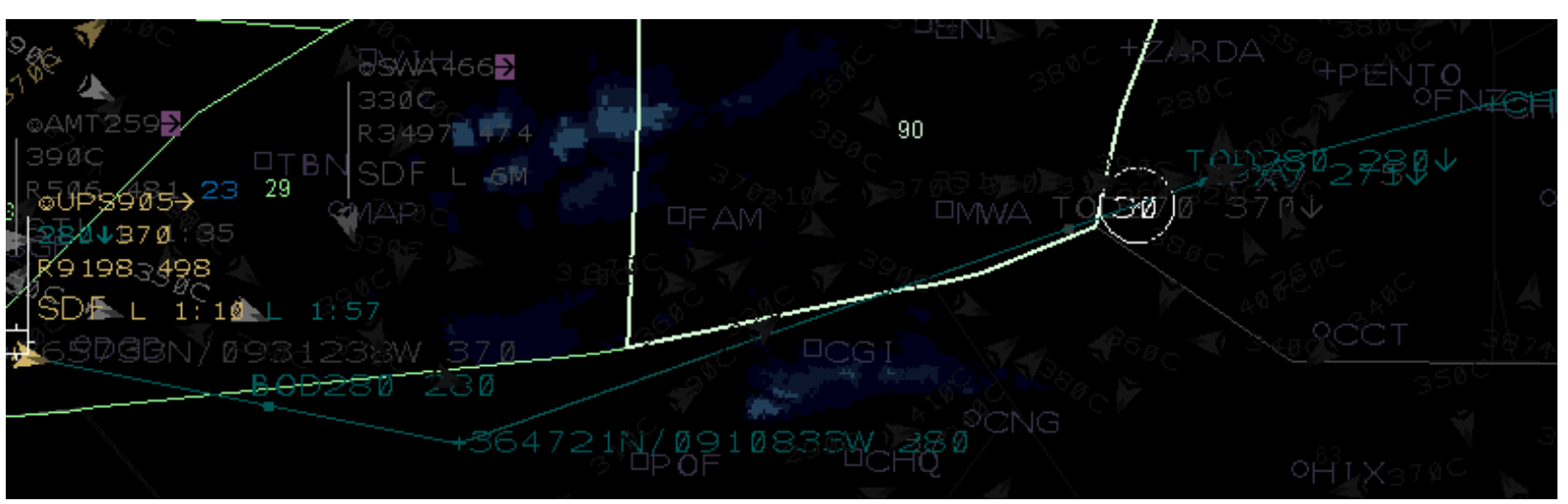

Figure A3. "TA" command showed the new route at the specified altitude (FL370 to FL280).

\section{TR (route specific trial planning)}

The TR command enabled the user to start a specific route trial plan. The planner would type the TR command followed by the waypoints they wanted for the aircraft and the ACID, and the trial plan route for that aircraft would appear on the DSR (Fig. A4). The trial plan line could then be graphically manipulated if needed. The TR function also worked interactively with the DSR scope by allowing the user to type TR, then click anywhere on the scope to build a new waypoint and then type ACID. The downstream portion of the original route remained unchanged.

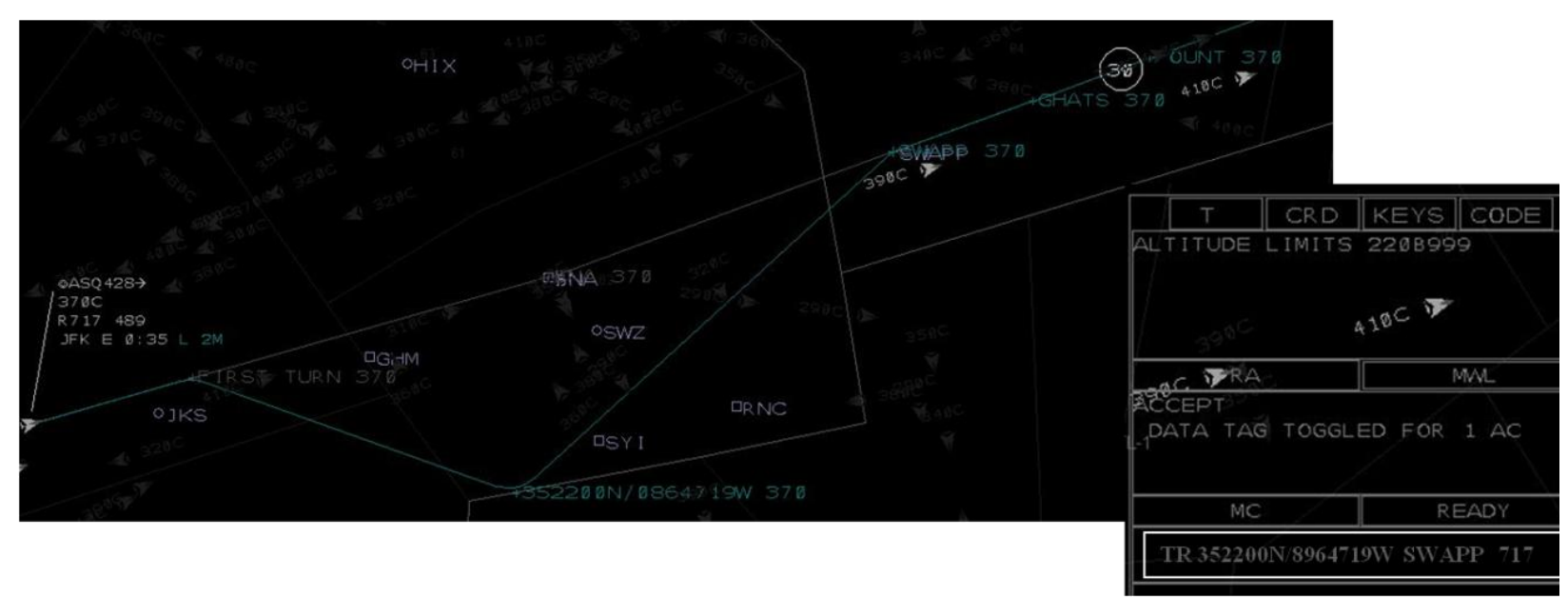

Figure A4. "TR" command enabled the user to graphically manipulate the route.

5. Flight Data Block Trial Plan Portals (arrow, magenta -arrow, altitude, weather number, and conflict number)

The FDB portals require picking on specific portions of the FDB. The basic trial plan portal is the arrow located to the right of the aircraft call sign and it functioned like a TT command. The arrow opened basic route trial plan with the drop down fix box and cyan route of flight. The trial plan could then be changed as the user wished.

The arrow turned a magenta color when there was a suggested trial plan from another planner or after the user had sent a trial plan via data link to another user. To review a suggested trial plan sent from another planner or to see a trial plan the user had already sent, picking on the magenta arrow displayed the route in magenta.

The altitude portion of the FDB started an altitude trial plan. This differs from a TA in that the user then selects an altitude to trial plan from a drop down list (Fig. A5) as opposed to typing the altitude. 


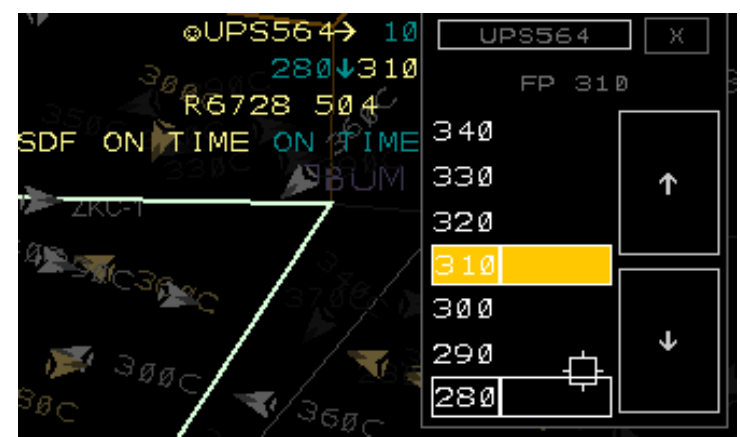

Figure A5. An altitude selection window appeared after picking on the altitude in the datablock.

Another way to open a trial plan via the FDB was with the weather or conflict number. The weather number showed how many minutes until the aircraft was predicted to penetrate weather (Fig. A2) and is located in the third line of the FDB next to the indicated airspeed. The conflict number was located to the right of the trail plan portal arrow and showed how many minutes until the aircraft will be in potential conflict with another aircraft (Fig. A5). Picking on these numbers opened a trial plan in order for the user to solve for the conflict or the weather penetration problem.

\section{References}

1 Corker, K., Liang, D., Lee, P., and Prevot, T., "New Air Traffic Management Concepts Analysis Methodology: Application to a Multi-Sector Planner," US Airspace Air Traffic Control Quarterly, Vol. 15, No. 4, 2007, pp. 347-367.

2 Smith, N., Lee, P. U., Prevot, T., Homola, J., Lee, H., Kessell, A., Brasil, C., Mainini, M. and Mercer, J. (2010) A Humanin-th-Loop Investigation of Multi-Sector Planning Operations for the NextGen Mid-Term, 10th AIAA AviATION TECHNOLOGY, INTEGRATION, AND OPERATIONS (ATIO) CONFERENCE, FORT WORTH, TX., 2010.

3 Eurocontrol En Route Multi-Sector Planning procedures. (PHARE/EHQ/PAT-7.5.2.1;1.1). Brussels, Belgium, 1998.

4 Herr, S., Tiechmann, M., Poppe, M. and Suarez, N. The Impact of Multi-Sector Planning and New Working Procedures on Controller Tasks. In Proceedings of the 24th AIAA/IEEE Digital Avionics Systems Conference (DASC), Washington, DC., 2005.

5 Prevot T, Smith N, Palmer E, Mercer J, Lee P, Homola J, and Callantine T. The Airspace Operations Laboratory (AOL) at NASA Ames Research Center, AIAA-2006-6112, AIAA, Reston, VA, 2006.

6 Prevot T, Lee P, Callantine T, Mercer J, Homola J, Smith N, et al. (in press). Human-in-the-Loop Evaluation of NextGen Concepts in the Airspace Operations Laboratory. Proceedings of the AIAA Modeling and Simulation Technologies (MST) Conference, Toronto, Canada, 2010.

7 Prevot T. Exploring the Many Perspectives of Distributed Air Traffic Management: The Multi-Aircraft Control System MACS. In S. Chatty, J. Hansman, \& G. Boy. (Eds). HCI-Aero 2002, AIAA Press, Menlo Park, CA. pp 149-154, 2002.

8 FAA (2010) NAS Enterprise Architecture Portal https://nasea.faa.gov

9 Prevot, T, Lee P., Martin L., Mercer J., Palmer E., \& Smith N. (2006) Tools for Trajectory-Based Air Traffic Control and Multi Sector Planning, HCI-Aero 2006, Seattle, WA, September 2006

10 Prevot T. NextGen Technologies for Mid-Term and Far-Term Air Traffic Control Operations. $28^{\text {th }}$ Digital Avionics Systems Conference, Orlando FL, 2009

11 Mercer J, Prevot T, Brasil C, Mainini M, Kupfer M, and Smith N. An integrated tool suite for en route radar controllers in NextGen. Proc $27^{\text {th }}$ International Congress of the Aeronautical Sciences, Nice, France, 2010. 